\title{
Hexagon bootstrap in the double scaling limit
}

\author{
Vsevolod Chestnov $^{a}$ and Georgios Papathanasiou ${ }^{b}$ \\ ${ }^{a}$ II. Institut für Theoretische Physik, Universität Hamburg, \\ Luruper Chaussee 149, 22761 Hamburg, Germany \\ ${ }^{b}$ DESY Theory Group, DESY Hamburg, \\ Notkestraße 85, D-22603 Hamburg, Germany \\ E-mail: vsevolod.chestnov@desy.de, georgios.papathanasiou@desy.de
}

Abstract: We study the six-particle amplitude in planar $\mathcal{N}=4$ super Yang-Mills theory in the double scaling (DS) limit, the only nontrivial codimension-one boundary of its positive kinematic region. We construct the relevant function space, which is significantly constrained due to the extended Steinmann relations, up to weight 13 in coproduct form, and up to weight 12 as an explicit polylogarithmic representation. Expanding the latter in the collinear boundary of the DS limit, and using the Pentagon Operator Product Expansion, we compute the non-divergent coefficient of a certain component of the Nextto-Maximally-Helicity-Violating amplitude through weight 12 and eight loops. We also specialize our results to the overlapping origin limit, observing a general pattern for its leading divergences.

Keywords: Scattering Amplitudes, Supersymmetric Gauge Theory

ArXiv EPRINT: 2012.15855 


\section{Contents}

1 Introduction 1

2 The six-gluon amplitude in the double-scaling limit 4

2.1 Analytic structure of the normalized amplitude 4

2.2 The double-scaling (DS) limit 8

$\begin{array}{lll}2.3 & \text { Integrability and extended Steinmann conditions } & 10\end{array}$

$\begin{array}{ll}2.4 \text { Branch cut conditions and transcendental constants } & 11\end{array}$

3 Bootstrapping the DS functions $\quad 13$

3.1 Solving the integrability and extended Steinmann constraints 13

$\begin{array}{lll}3.2 & \text { Promoting coproducts to functions } & 16\end{array}$

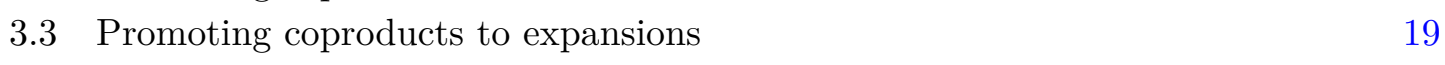

4 The Wilson loop OPE and the collinear limit 21

4.1 Wilson loop normalization, variables and $R$-invariants in the DS limit 21

4.2 NMHV Wilson loop OPE 23

5 Results $\quad \mathbf{2 8}$

5.1 The NMHV amplitude up to 8 loops and weight $12 \quad 28$

$\begin{array}{ll}5.2 \text { The origin limit } & 29\end{array}$

5.3 Further refinements of the $\mathcal{H}^{\mathrm{DS}}$ space 31

A The multiple zeta value basis $\quad 32$

B Tensor perspective on the coproduct bootstrap 33

\section{Introduction}

The exact description of quantum interactions is one of the outstanding questions in theoretical physics. Our best hope for answering this question is in the realm of the simplest interacting quantum field theory in four dimensions, $\mathcal{N}=4$ super Yang-Mills theory (SYM) $[1,2]$. Indeed, in the large-color or planar limit [3] the integrability of the theory has enabled the determination of the scaling dimension spectrum of all its single-trace operators beyond perturbation theory, see for example the reviews [4, 5].

For the quantities actually encoding the outcome of particle interactions, namely the scattering amplitudes, which in this theory also happen to be equivalent to null polygonal Wilson loops [6-8], integrable structures are currently known to emerge only in certain corners of the space of kinematics. The best-understood such corner is a certain collinear limit [9], whereby every term in the series expansion of the Wilson loop or amplitude with 
respect to the kinematic variables that become small in this limit, can be mapped to an exactly solvable flux tube [10-19]. The flux tube description, known as the Wilson loop (or Pentagon) Operator Product Expansion (OPE), is complete for the first nontrivial amplitude of the theory, which as a consequence of dual conformal symmetry (see [20] for a review) has multiplicity $n=6 .^{1}$

In order to obtain closed expressions for this six-particle or 'hexagon' amplitude, which will be the focus of this article, both at finite coupling and in general kinematics, one would thus have to resum the aforementioned kinematic expansion. A strategy to achieve this ambitious goal, would be to divide it into two simpler steps: first resum the kinematics order by order in perturbation theory with respect to the planar coupling $g$, and then resum the perturbative series. ${ }^{2}$ Indeed, a great deal is known about the class of polylogarithmic functions the first step evaluates to, thus greatly facilitating its realization. And it is not unreasonable to expect that these polylogarithms can be in turn resummed to more complicated functions of hypergeometric type, as has been the case with certain integrals contributing to the amplitude [26], thanks to the existence of differential equations relating different perturbative orders.

The task of resumming the perturbative OPE series was initiated in $[27],{ }^{3}$ also building on the earlier work $[32,33]$, under one additional simplification: starting from the onedimensional collinear limit, the kinematics was resummed to the "double-scaling" (DS) limit $[13,34]$, instead of the full three-dimensional space of general kinematics of the hexagon. The double-scaling limit is distinguished by the fact that it is the only codimension-one boundary of the region of positive kinematics, where the (appropriately normalized [35]) amplitude is nonvanishing, modulo its discrete symmetries. The positive region, first considered in the context of amplitude integrands [36] and then adapted to the space of external kinematics in [37], is part of the Euclidean region, where amplitudes are free of branch points. Indeed, as is reviewed in e.g. [38], the only other codimension-one boundary is the soft or equivalently multi-Regge limit, where the amplitude is nonvanishing only after analytically continuing away from the Euclidean region.

From the point of view of the flux tube description, the double scaling limit is advantageous because only a simpler subset, of so-called same-helicity gluon excitations contribute. These are not charged under the internal symmetries of the theory, and are labeled by a particle number $N$, which also corresponds to the dimensionality of their all-loop integral representation. In [27], in was in particular the $N=1$ excitations which were considered, and it was realized that existing nested summation algorithms [39] allow their explicit evaluation in terms of two-dimensional harmonic polylogarithms [40], or more precisely their subset associated to the $A_{2}$ cluster algebra [37], in principle at any loop order.

In this work, we take the next step and study the $N=2$ gluon OPE excitations. These give rise to significantly more complicated integrals where, to the best of our knowledge, no direct method for their evaluation is available to date (see [41] for the current state of the

\footnotetext{
${ }^{1}$ At higher multiplicity, one final building block known as the "matrix part" is still missing.

${ }^{2}$ Alternatively, one could start from the strong- instead of the weak-coupling regime, see [21-25] for work in this direction.

${ }^{3}$ For more recent work on the weak-coupling OPE resummation, see also [28-31].
} 
art with respect to nested summation technology), despite the fact that they are expected to lie in the same space of functions as the $N=1$ excitations. Instead, we will rely on the bootstrap philosophy, where one first constructs the expected space of functions, and then locates the physical quantity in question within this space. This approach has been first applied in the similar setting of the multi-Regge limit in [42], and more recently it has been very successful for determining perturbative six- and seven-particle amplitudes in planar $\mathcal{N}=4 \mathrm{SYM}$ in general kinematics [43-55]. In particular, the former are known through six and seven loops in the Next-to-Maximally Helicity-Violating (NMHV) and Maximally-Helicity-Violating (MHV) configuration, respectively, whereas the latter are known through four loops, see also the recent review [56].

First, we will thus develop the hexagon bootstrap in the simplified setting of the double scaling limit. We will see that while the limit breaks some of the symmetries of the amplitude, it still preserves important analytic properties that tame the growth of the space of relevant functions with respect to the weight, namely the number of iterated integrations defining them. Among these properties, a special role will be played by the extended Steinmann relations [26, 54], which generalize the ordinary Steinmann relations [57-59] so as to forbid not only double, but also multiple discontinuities in overlapping channels. While these channels are normally associated to Mandelstam invariants, for functions with physical branch cuts the extended Steinmann relations imply the absence of discontinuities also with respect to more general kinematic variables, as also predicted by the principle of cluster adjacency $[38,60]$. Specifically in the double-scaling limit, we find that two of such generalized discontinuities are forbidden. With their aid, we will be able to construct the corresponding space of 'Extended Steinmann Double-Scaling' (DS for short) functions to weight 12 explicitly, and to weight 13 when the functions are specified iteratively in terms of their first derivatives (or coproducts, see [61] for a review).

Then, we will proceed to uniquely identify the contribution of $N=1,2$ gluon OPE excitations inside the DS space. On the one hand, we will work out the expansion of our functions in the collinear limit, and on the other hand we will compare that to the sum representation of the OPE predictions, obtained from their original integral form with the help of Cauchy's residue theorem, and organized into finite coefficients multiplying divergent logarithms in the limit. So as to be able to provide useful boundary data and checks to the amplitude bootstrap in general kinematics, here we will be focusing on the contributions to the NMHV (super)amplitude, which carries both rational and transcendental dependence on the kinematics. Given that the $N>3$ OPE contributions only start contributing at higher loop orders, in this manner we will be able to determine the finite coefficients in the DS limit of the NMHV hexagon for a particular, so-called (1111) component of its rational dependence through weight 12 and eight loops.

With these results at hand, it is possible to study further interesting subspaces of the DS limit. As an example, we will indeed also specialize them to a DS boundary point known as the origin limit [53], where a similar OPE resummation strategy as the one employed here, has led to finite-coupling conjectures for the form of the MHV amplitude [62], which exhibits a Sudakov-like exponentiation. As was observed in the latter paper, and we confirm here, the NMHV amplitude no longer exhibits this exponentiation. Nevertheless, we observe a general pattern for its leading divergence at the origin, which may be valid to all loops. 
Finally, we briefly address the question of how 'minimal' the DS function space is, namely whether it also contains redundant functions which are not present in the amplitude or its derivatives. By comparing with the space spanned by the latter, we notice that nonconstant redundant functions already start appearing at weight three. Understanding the reason for this redundancy, and further refining our space by eliminating it, are interesting questions that we leave for the future. Perhaps more importantly, in follow-up work we look forward to using the host of explicit results we have obtained, in order to develop new direct evaluation algorithms for the two-gluon OPE contributions, which may also be more broadly applicable to perturbative quantum field theory. The presence of a similar successful paradigm, where the knowledge of certain double pentagon ladder integrals [63] to high loop order [26] subsequently led to new computational methods [64], is very encouraging in this respect.

This paper is organized as follows. In section 2, we start by reviewing the essential analytic properties of the six-particle amplitude in general kinematics, and then move on to work out their implications in the DS limit. Relying on these properties, in section 3 we describe the construction of our DS function space and its expansion in the collinear limit. The latter is to be compared with the predictions of the Wilson loop OPE, discussed in section 4. The thus obtained new results on the on the NMHV amplitude in the DS limit are presented in section 5. Finally, we have included two appendices with further details on our DS space construction. Our results are also attached as computer-readable files accessible at [65].

\section{The six-gluon amplitude in the double-scaling limit}

In this section we deduce the analytic properties of the six-particle amplitude in the DS limit. In subsection 2.1 we first review some background information on the normalization of the amplitude, its analytic structure in general kinematics, and the class of multiple polylogarithms encoding it. Then in subsection 2.2 we define the DS limit, and describe the potential amplitude singularities in the limit. Subsection 2.3 continues with the analysis of the property of integrability and of the extended Steinmann relations, especially illustrating the constraining power of the latter. Finally in subsection 2.4 we derive additional conditions obeyed by the amplitude and its derivatives in the DS limit, stemming from the absence of unphysical branch cuts.

\subsection{Analytic structure of the normalized amplitude}

Let us start by reviewing some known facts about the normalization, kinematic dependence and analytic properties of the planar six-particle amplitude in $\mathcal{N}=4 \mathrm{SYM}$. This will also serve for establishing our conventions, which are mostly adapted from the review [56]. Readers familiar with this material may skip to the next section.

The infrared divergence structure of the six-particle amplitude is well-understood [35] and can be factored out, giving rise to a finite normalized amplitude. Different conventions on this normalization reflect the freedom to also absorb finite terms in this infrared-divergent

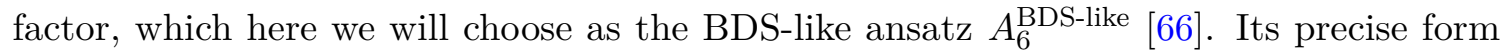


will not be important for our purposes, and instead we will be focusing on the BDS-like normalized amplitudes of the two inequivalent helicity configurations,

$$
\mathcal{E}:=\frac{A_{6, \mathrm{MHV}}}{A_{6, \mathrm{MHV}}^{(0)} A_{6}^{\mathrm{BDS}-\text { like }}}, \quad \mathrm{E}:=\frac{A_{6, \mathrm{NMHV}}}{A_{6, \mathrm{MHV}}^{(0)} A_{6}^{\mathrm{BDS}-\text { like }}},
$$

where we have also divided out by their known tree-level contribution. While $\mathcal{E}$ is given just by a single bosonic function of the kinematics, E may in turn be further decomposed as

$$
\begin{aligned}
\mathrm{E}= & \frac{1}{2}[((1)+(4)) E(u, v, w)+((2)+(5)) E(v, w, u)+((3)+(6)) E(w, u, v) \\
& +((1)-(4)) \tilde{E}(u, v, w)+((2)-(5)) \tilde{E}(v, w, u)+((3)-(6)) \tilde{E}(w, u, v)] .
\end{aligned}
$$

where $(1):=[23456]$ and its cyclic permutations denote the so-called $R$-invariants $[63,67$, 68], namely rational terms of the kinematics and the Graßmann variables, encoding the superconformal and dual superconformal symmetry of the amplitude.

The two functions $E$ and $\tilde{E}$ introduced in eq. (2.2), as well as the entire MHV amplitude $\mathcal{E}$, are bosonic functions of the kinematical data, which can be conveniently parametrised by a set of three cross-ratios:

$$
\{u, v, w\}:=\left\{\frac{s_{12} s_{45}}{s_{123} s_{345}}, \frac{s_{23} s_{56}}{s_{234} s_{123}}, \frac{s_{34} s_{61}}{s_{345} s_{234}}\right\}
$$

which are invariant under the parity transformation. Evidence from all explicit results to date, as well as from the analysis of the integrand [69] (note however the caveats pointed out in [70]) implies that the order $g^{2 L}$ ( $L$-loop) contribution in the weak coupling expansion of $\mathcal{E}, E, \tilde{E}$ can be expressed in terms of multiple polylogarithms (MPLs) [71-74] (see also the review [61]) of transcendental weight $p=2 L$. A function $F^{(p)}$ is said to be an MPL of weight $p$ if its total differential obeys

$$
d F^{(p)}=\sum_{\beta \in \mathcal{A}}\left(F^{(p-1)}\right)^{\beta} d \log (\beta),
$$

such that $\left(F^{(p-1)}\right)^{\alpha}$ is an MPL of weight $(p-1)$ and so on, where the recursive definition terminates with the usual logarithms $(p=1)$ on the left-hand side, and rational numbers $(p=0)$ as coefficients of the total differentials on the right-hand side. The set $\mathcal{A}$ of arguments of dlog forms is called the symbol alphabet, and it encodes positions of possible branch points of the $F^{(p)}$ function.

This recursive nature of the differential of MPLs is a part of a deeper structure revealed by the coproduct (more precisely, coaction) $\Delta$ [75-78], which, very roughly, decomposes an MPL of weight $p$ into a sum of tensor products of MPLs of lower weight. ${ }^{4}$ In particular, the total differential (2.4) is essentially equivalent to the $\{p-1,1\}$ component of $\Delta$,

$$
\Delta_{p-1,1} F^{(p)}=\sum_{\beta \in \mathcal{A}}\left(F^{(p-1)}\right)^{\beta} \otimes[\log (\beta) \bmod (\mathrm{i} \pi)] .
$$

\footnotetext{
${ }^{4}$ Strictly speaking, this decomposition is conjectural, principally due to a lack of formal proof that there exist no identities between MPLs of different weight. In the following we will assume this well-tested conjecture to hold and refer the interested reader to [61, 78] for further discussions of this point.
} 
Further considering the total differential of $F^{(p-1)}$, or equivalently the analogue of eq. (2.5) for the latter, then yields the $\{p-2,1,1\}$ component of the coproduct,

$$
\Delta_{p-2,1,1} F^{(p)}=\sum_{\alpha, \beta \in \mathcal{A}}\left(F^{(p-2)}\right)^{\alpha, \beta} \otimes \log \alpha \otimes \log \beta,
$$

where we will also refer to the leftmost factor on the right-hand side as the 'double coproduct'. In the above formula, and in what follows, identification of logarithmic factors up to $\mathrm{i} \pi$ is implied in all but the first slot of the tensor product. We can also continue the decomposition of the leftmost coproduct factor, until we reach the maximal, $p$ times iterated coproduct $\{1, \ldots, 1\}$, which is also known as the symbol.

Another very usefull point of view on the MPLs stems from their integral representation: an MPL is defined to be a $\mathbb{Q}$-linear combination of the following iterated integrals (sometimes referred to as "hyperlogarithms"):

$$
G\left(a_{1}, \ldots, a_{p} ; z\right):=\int_{0}^{z} G\left(a_{2}, \ldots, a_{p} ; t\right) \frac{\mathrm{d} t}{t-a_{1}}, \quad G(; z):=1,
$$

where the special case of only zero arguments is covered by this rule:

$$
G(\underbrace{0, \ldots, 0}_{p} ; z):=\frac{(\log z)^{p}}{p !} .
$$

The space of so-called hexagon functions containing $\{\mathcal{E}, E, \tilde{E}\}$ and their coproducts is in fact a much smaller subspace of all MPLs, due to additional physical and mathematical constraints that the amplitude satisfies. The idea of the hexagon function bootstrap [43$47,49,50,53,54]$ is to first construct this space from its basis, then form an ansatz for the amplitude, and finally find a unique solution for the latter by comparing it to the behavior of the amplitude in various kinematic limits, known by other means. Below we will briefly review the additional analytic properties of hexagon functions, which we will then specialize to the double-scaling limit.

Symbol alphabet. The space of hexagon functions containing $\{\mathcal{E}, E, \tilde{E}\}$ are MPLs as defined in eq. (2.4), whose letters are drawn from the following $\operatorname{list}^{5}[43]$ :

$$
\mathcal{A}^{\text {hex }}=\left\{u, v, w, 1-u, 1-v, 1-w, y_{u}, y_{v}, y_{w}\right\} .
$$

Apart from the parity-even cross ratios of eq. (2.3), we have also introduced parity odd letters that are expressed in terms of the latter $\left(u_{1}=u, u_{2}=v, u_{3}=w\right.$ and similarly for $\left.y_{i}\right)$ as

$$
y_{i}:=\frac{u_{i}-z_{+}}{u_{i}-z_{-}}, \quad z_{ \pm}:=\frac{1}{2}(-1+u+v+w \pm \sqrt{\delta}), \quad \delta:=(1-u-v-w)^{2}-4 u v w .
$$

\footnotetext{
${ }^{5}$ The general hexagon alphabet was derived at 2 loops by an explicit computation in $[79,80]$ as well as in the analysis of related integrals in $[81,82]$. Up to now, all the available evidence suggests that it does not change at higher perturbative orders.
} 
Zeros of these expressions label the possible locations of the branch cut singularities of MPLs in the ansatz.

First entry condition. Locality dictates that in the Euclidean region, amplitudes can only develop singularities at its boundary, where any of the cross ratios tend to zero. This implies that only the first three letters in eq. (2.9) are allowed to appear in the first entry of the symbol [34], or equivalently that the weight-one space of hexagon functions consists of

$$
F^{(1)} \in\{\log u, \log v, \log w\} .
$$

Integrability conditions. Any well-defined hexagon function $F$ must satisfy

$$
\frac{\partial^{2} F}{\partial x_{i} \partial x_{j}}=\frac{\partial^{2} F}{\partial x_{j} \partial x_{i}}, \quad i \neq j
$$

for any choice of variables $x_{1}, x_{2}, x_{3}$ parametrizing the kinematics, such as the cross ratios (2.3). Given the relation between total differentials and coproduct components (2.4) and (2.5), these translate to linear relations for the left factors of the double coproduct of eq. (2.6), known as the integrability conditions. For hexagon functions it's easy to show that there are 26 such relations, whose explicit form in the particular choice of alphabet (2.9) may be found e.g. in [49].

Extended Steinmann relations. Basic principles of quantum field theory prohibit virtual particles in any physical process to simultaneously become on-shell in two overlapping channels [57-59]. This can be translated into the vanishing of certain double discontinuities of the associated physical quantity, or equivalently into restrictions on the first two entries of its symbol (if it is described by MPLs). In $[26,54]$ it was realized that these relations in fact hold not on just the first two, but on any adjacent letters in the symbol. These 'extended Steinmann relations' may be equivalently be stated in terms of the double coproducts of any hexagon function $F$ as

$$
F^{v, u}+F^{v, w}+F^{w, u}+F^{w, w}=0,
$$

together with two other cyclic permutations $u \rightarrow v \rightarrow w \rightarrow u$. When combined with the other analytic properties mentioned thus far, they also automatically imply the following double coproduct relations,

$$
\begin{aligned}
F^{1-u, v}+F^{1-u, w}+F^{1-u, 1-v}+F^{1-u, 1-w} & =0 \\
F^{w, y_{w}}+F^{1-v, y_{w}}+F^{1-w, y_{w}}-F^{v, y_{v}}-F^{1-v, y_{v}}-F^{1-w, y_{v}}+F^{v, y_{w}}-F^{w, y_{v}} & =0 \\
F^{1-u, y_{v}}-F^{1-u, y_{u}}+F^{y_{v}, 1-w}-F^{y_{w}, 1-w} & =0 \\
F^{1-v, 1-u}+F^{y_{u}, y_{v}}+F^{y_{w}, y_{w}}-F^{y_{u}, y_{w}}-F^{y_{w}, y_{v}} & =0
\end{aligned}
$$

plus cyclic permutations. The above relations, eqs. (2.13)-(2.17), on integrable functions are also predicted by the principle of cluster adjacency $[38,60]$, which relies on the fact that the letters (2.9) are isomorphic to the variables of a mathematical object known as $A_{3}$ cluster algebra [37]. Part of the structure of this object is the arrangement of the cluster variables in overlapping sets, which may be naturally translated to double coproduct relations. 
Branch cut conditions and transcendental constants. All of the above double coproduct relations in fact define hexagon functions only up to constants times weight-one logarithms. Not every value of these constants is allowed however, since it may lead to beyond-the-symbol terms with unphysical branch cuts. As was discussed in [45, 49], one way to eliminate this possibility is to additionally impose that hexagon functions are well-behaved in certain kinematic limits.

Here we will adopt the choice of the aforementioned papers, and consider the soft limit

$$
u, w \rightarrow 0, v \rightarrow 1 \text { with } \frac{u}{1-v}, \frac{w}{1-v} \text { fixed } \Leftrightarrow y_{v} \rightarrow 1 \text { with } y_{u}, y_{w} \text { fixed }
$$

where the $\{p-1,1\}$ coproduct component of hexagon functions should obey

$$
\left.F^{1-v}\right|_{y_{v} \rightarrow 1}=\left.F^{y_{u}}\right|_{y_{v} \rightarrow 1}=\left.F^{y_{w}}\right|_{y_{v} \rightarrow 1}=0 .
$$

These conditions need to also be supplemented with the set of transcendental constants that we include as independent functions in our basis, and then they determine the precise rational coefficients these multiply weight-1 logarithms with. As is also reviewed in [56], on general grounds these constants should be drawn from multiple zeta values (MZV), and in $[53,54]$ it was furthermore conjectured that only their subset of even ordinary zeta values of weight at least four,

$$
\left\{\zeta_{4}, \zeta_{6}, \zeta_{8}, \zeta_{10}, \zeta_{12}, \ldots\right\}
$$

is necessary for the six-particle amplitude and its derivatives at any loop order.

Let us now proceed to work out the consequences of these analytic properties of the amplitude in the double-scaling limit, which will be the focus of this article.

\subsection{The double-scaling (DS) limit}

The double-scaling limit $[13,27,34]$ of the six-particle amplitude in the planar $\mathcal{N}=4$ SYM is generally defined as the limit where one of the three cross ratios (2.3) goes to zero, whereas the remaining two are held fixed. In this work we will choose the particular orientation

$$
\text { DS limit: } w \rightarrow 0 \text { with } u, v \text { fixed, }
$$

where it is easy to show that the discriminant of eq. (2.10) reduces to

$$
\sqrt{\delta} \underset{\mathrm{DS}}{\longrightarrow} \pm(1-u-v)\left[1-\frac{(1-u-v+2 u v) w}{(1-u-v)^{2}}\right]+\mathcal{O}\left(w^{2}\right)
$$

with different signs denoting the two possible choices of the square root branch, such that the odd letters become

$$
y_{u} \rightarrow\left[\frac{u}{1-v}\right]^{ \pm 1}, \quad y_{v} \rightarrow\left[\frac{v}{1-u}\right]^{ \pm 1}, \quad y_{w} \rightarrow\left[\frac{(1-u)(1-v) w}{(1-u-v)^{2}}\right]^{ \pm 1}
$$

Comparing with eq. (2.9), we thus see that in this limit hexagon functions $F$ reduce to divergent logarithms in $w$, times 'DS functions' $f$ with alphabet

$$
\{u, v, 1-u, 1-v, 1-u-v\}
$$




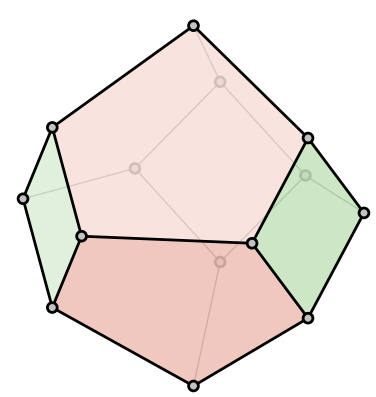

Figure 1. The $A_{3}$ Stasheff associahedron, relevant for six-particle scattering. The positive region in the space of kinematics amounts to the interior of the polytope, whereas pentagonal faces correspond to double-scaling limits, and square faces to soft (or equivalently multi-Regge) limits. The two red pentagonal faces represent the two possible double scaling limits DS1 and DS2 that are considered in this work.

By virtue of eq. (2.23), the $\{p-1,1\}$ coproducts of the DS functions are related to the hexagon functions $F$ as follows:

$$
\begin{aligned}
f^{u} & =F^{u} \pm F^{y_{u}}, \\
f^{v} & =F^{v} \pm F^{y_{v}} \\
f^{1-u} & =F^{1-u} \pm\left(F^{y_{w}}-F^{y_{v}}\right), \\
f^{1-v} & =F^{1-v} \pm\left(F^{y_{w}}-F^{y_{u}}\right), \\
f^{1-u-v} & =\mp 2 F^{y_{w}} .
\end{aligned}
$$

(plus $f^{\prime w}=F^{w} \pm F^{y w}$ if $f^{\prime}$ denotes the products of $f$ with $\log w$ ). As it was with eq. (2.22), the \pm sign here is due to the fact that hexagon functions are in fact well-defined only in the double cover of the cross-ratio parametrization of the kinematics. In other words, there really exist two $w \rightarrow 0$ double-scaling limits DS1 and DS2, that are related by a parity transformation, $y_{i} \rightarrow 1 / y_{i}$. With the appropriate choice of independent kinematic variables, where the region of positive kinematics is the particular blowup of the unit cube shown in figure 1, the two DS limits are the boundaries depicted in red. As is reviewed in e.g. [38], the six-particle positive region has the topology of the Stasheff associahedron, closely related to the $A_{3}$ cluster algebra, whereas pentagonal faces represent its $A_{2}$ subalgebras.

In what follows, we will use an alphabet equivalent to (2.24), which arises naturally when evaluating the Wilson loop OPE contributions to the DS limit. In particular, we define

$$
x:=-\frac{1-u-v}{v}, \quad y:=\frac{1-v}{1-u-v},
$$

or conversely

$$
u=\frac{x(1-y)}{1-x y}, \quad v=\frac{1}{1-x y},
$$

such the alphabet (2.24) takes the equivalent form

$$
\mathcal{A}:=\{x, y, 1-x, 1-y, 1-x y\},
$$


which we will adopt from this point on. For completeness, we also quote how the $\{p-1,1\}$ coproducts of the DS functions in the two equivalent alphabets are related by virtue of eq. (2.26),

$$
\begin{aligned}
f^{u} & =f^{1-y}, \\
f^{v} & =-f^{x}-f^{1-x}-f^{1-x y}, \\
f^{1-u} & =f^{1-x}, \\
f^{1-v} & =f^{y} \\
f^{1-u-v} & =f^{x}-f^{y}-f^{1-y} .
\end{aligned}
$$

Finally, in the above choice of alphabet, it is evident that for DS functions, the first entry condition (2.11) becomes

$$
f^{(1)} \in\{\log (1-x y), \log (x(1-y))\} .
$$

\subsection{Integrability and extended Steinmann conditions}

After defining the DS limit, in the previous subsection we also analyzed the symbol letters and first entry condition of the space of functions expected to capture six-particle scattering in this limit. Here, we continue by deriving constraints on the double coproducts of these functions.

First, the integrability conditions are very simple to derive for the alphabet (2.28) we will be using from a single equation of the form (2.12), with $x_{1}=x$ and $x_{2}=y$. It gives rise to the following set of six equations,

$$
\begin{aligned}
f^{x, y}-f^{y, x}=f^{1-x, y}-f^{y, 1-x}=f^{x, 1-y}-f^{1-y, x} & =0, \\
f^{1-x, 1-x y}+f^{1-x, 1-y}-f^{1-x y, 1-x}-f^{1-y, 1-x} & =0, \\
f^{1-x, 1-y}+f^{1-x y, 1-y}-f^{1-y, 1-x}-f^{1-y, 1-x y} & =0, \\
f^{x, 1-x y}-f^{1-x, 1-y}-f^{1-x y, x}+f^{1-x y, y}-f^{y, 1-x y}+f^{1-y, 1-x} & =0 .
\end{aligned}
$$

Alternatively, the above DS integrability conditions may be obtained from those in general six-particle kinematics, discussed in section 2.1, as follows: they are the subspace of 6 out of the 26 integrability conditions in general kinematics, that only depend on the particular double coproduct combinations that are induced by eq. (2.25).

Next, we examine the extended Steinmann relations, as well as the additional relations they imply on integrable functions with the alphabet (2.9) and first entries (2.11), eqs. (2.13)(2.17), following the same procedure for obtaining the integrability conditions in the DS limit from those in general kinematics. Namely we look for the subspace of these 15 equations, that is spanned by only the linear combinations of double coproducts that are present in the DS limit. In this manner we obtain another two equations on top of eqs. (2.31),

$$
f^{1-x, y}=f^{x, 1-x y}+f^{1-x, 1-x y}+f^{1-x y, 1-x}+f^{1-x y, 1-x y}+f^{1-x y, 1-y}-f^{y, 1-x y}=0 .
$$

Owing to their origin in general kinematics, we will denote eqs. (2.32) as the 'DS Extended Steinmann relations'. Note that the procedure we have described for reducing any set of 


\begin{tabular}{|l|ccccccccccccc|}
\hline weight $p$ & 1 & 2 & 3 & 4 & 5 & 6 & 7 & 8 & 9 & 10 & 11 & 12 & 13 \\
\hline First entry & 2 & 5 & 13 & 35 & 97 & 275 & 793 & 2315 & 6817 & 20195 & 60073 & 179195 & 535537 \\
Ext. Stein. & 2 & 4 & 9 & 19 & 39 & 78 & 154 & 302 & 591 & 1157 & 2269 & 4460 & 8788 \\
\hline
\end{tabular}

Table 1. The dimension of the space of symbols with physical branch cuts in the DS limit (first line), that additionally obey the extended Steinmann relations (second line).

linear equations from general to special kinematics is completely systematic, ${ }^{6}$ hence there is no ambiguity or room for confusion with the application of the extended Steinmann relations, even though different hexagon symbol letters may become linearly dependent in the DS limit: assuming the space of extended Steinmann hexagon functions contains the six-particle amplitude in general kinematics, this then implies that its DS limit will always be contained in the space of DS functions we construct here.

One may be tempted to think that the DS Extended Steinmann relations may only marginally reduce the size of the relevant function space, due to their relatively small number. As we see in table 1 however, their effect is in fact very significant: focusing momentarily on the space of symbols, i.e. polylogarithmic functions modulo transcendental constants (these will be reinstated in the next subsection) with the alphabet (2.28), in the first line we quote their number as a function of their weight $p$, when the weight-one or first entry space is constrained as dictated by eq. (2.30). We notice that this number grows by roughly a factor of three at each weight (more precisely, it is equal to $3^{p-1}+2^{p-1}$ ).

On the second line of table 1, we display how many of the functions of the first line, additionally obey the DS Extended Steinmann relations (2.32). Evidently, their number now grows by roughly a factor of two instead of three. Thus the DS Extended Steinmann relations are responsible for a massive reduction in the size of the relevant function space, for example by more than $98 \%$ for weight 13 . They will thus be pivotal for constructing this function space to high weight, as detailed in the next section, and for bootstrapping new results for the six-particle NMHV amplitude in the DS limit, as presented in section 5 .

This concludes the analysis of all symbol-level constraints on the DS functions space. In the next subsection, we discuss the additional constraints necessary to promote them to functions.

\subsection{Branch cut conditions and transcendental constants}

As briefly reviewed in subsections 2.1 and 2.2, hexagon or DS functions are allowed to have branch points only when a cross ratio approaches zero (or infinity). Focusing on DS functions, one should thus require that they are free of branch point singularities when $u \rightarrow 1, v \rightarrow 1$ or $u \rightarrow 1-v$, or equivalently that their derivatives are free of poles there.

\footnotetext{
${ }^{6}$ Equivalently, we could have considered the limit of the 'dual' space of weight-two functions appearing on the right-hand side of eq. (2.6). In particular, there are 40 weight-two functions of this sort in the extended Steinmann hexagon function space [54], whose $\log ^{0} w$ coefficients in the DS limit reduce to 17 functions. This in turn implies 8 linear relations between the double coproducts on the left-hand side of eq. (2.6) in the DS limit, which are precisely the six integrability conditions (2.31) together with the relations (2.32).
} 
Given that

$$
\frac{\partial f}{\partial u}=\frac{f^{u}}{u}-\frac{f^{1-u}}{1-u}-\frac{f^{1-u-v}}{1-u-v},
$$

the physical branch cut conditions thus translate to

$$
\left.f^{1-u}\right|_{u \rightarrow 1}=\left.f^{1-v}\right|_{v \rightarrow 1}=\left.f^{1-u-v}\right|_{u \rightarrow 1-v}=0 .
$$

Let us now translate the above branch cut limits and conditions in our choice of independent variables (2.26) and symbol letters (2.28). As far as the limits are concerned, we have

$$
\begin{aligned}
& u \rightarrow 1: x \rightarrow 1 \text { with } y \text { fixed, } \\
& v \rightarrow 1: y \rightarrow 0 \text { with } x \text { fixed, } \\
& u \rightarrow 1-v: x \rightarrow 0 \text { with } x y \text { fixed, }
\end{aligned}
$$

whereas by virtue of (2.29) the branch cut conditions become

$$
\left.f^{y}\right|_{y \rightarrow 0}=\left.f^{1-x}\right|_{x \rightarrow 1}=f^{x}-f^{y}-\left.f^{1-y}\right|_{\substack{x \rightarrow 0 \\ x y \text { fixed }}}=0 .
$$

A potential subtlety with the branch cut conditions (2.34) or (2.36), is that the limits they describe are not just the edges of the pentagonal faces of the Stasheff polytope depicted in figure 1, which are usually simpler to impose. This is especially the case with the third limit/condition in (2.36), which requires taking $y \rightarrow \infty$ simultaneously with $x \rightarrow 0$, and thus lies outside of the $0<x, y<1$ square, where any function with the alphabet $\mathcal{A}$ given in eq. (2.28) is free of branch cuts.

To avoid this complication, we will instead rely on the fact that the DS limit overlaps with the soft limit on the edge between a red pentagonal and a green square face of the Stasheff polytope in figure 1, where branch cut conditions for the general hexagon function space have been derived. From (2.27), it is clear that the DS limit intersects with the soft limit (2.18) for $x \rightarrow 0$ with $y$ fixed. Then, using (2.25) and (2.29) to relate the DS coproducts in the $\mathcal{A}$ alphabet to the hexagon function coproducts, and specializing to the double-scaling/soft overlap, we can show that by virtue of eq. (2.19),

$$
f^{x}-\left.f^{1-y}\right|_{x \rightarrow 0}=\left.F^{1-v} \mp\left(F^{y_{w}}+F^{y_{u}}\right)\right|_{y_{v} \rightarrow 1}=0 .
$$

Instead of eq. (2.36), we will thus choose to impose the following set of simpler branch cut conditions,

$$
\left.f^{y}\right|_{y \rightarrow 0}=\left.f^{1-x}\right|_{x \rightarrow 1}=f^{x}-\left.f^{1-y}\right|_{x \rightarrow 0}=0 .
$$

Finally, let us come to the question of which transcendental constants we should include in our DS function space as independent basis elements. While only the subset (2.20) of MZVs have been found to be necessary in general kinematics, it is easy to show that in the DS limit we will certainly also need $\zeta_{2}$. In particular, this is what the weight-two hexagon function $\operatorname{Li}_{2}(1-1 / w)=-G(0,1 ; 1-1 / w)$, see e.g. [54], reduces to in the limit. A similar analysis 
at weight three indicates that we also need to include $\zeta_{3}$ as an independent constant. Given that these low-weight constants will be responsible for the bulk of non-trivial functions with vanishing symbols in our space, we will choose to be agnostic and include all MZVs, a basis of which is contained in appendix A through weight twelve, as independent functions in our space. We will then come back and examine the possible redundancy of our space in the closing subsection 5.3.

Summarizing, we define our space of Extended Steinmann DS functions, $\mathcal{H}^{\mathrm{DS}}$, to consist of MPLs with the alphabet of eq. (2.28), whose first entry space is restricted to (2.30), and obeys the integrability (2.31) DS extended Steinmann (2.32) and branch cut conditions (2.38), also containing all MZVs as independent functions. In the next section, we describe the construction of this space in detail.

\section{Bootstrapping the DS functions}

The goal of this section is to construct a linear space of functions $\mathcal{H}^{\mathrm{DS}}$, which we refer to as the (Extended Steinmann) DS space, encoding six-particle scattering in the DS limit, defined in the previous section. The DS space should (conjecturally, see also footnote 4) have a grading by transcendental weight,

$$
\mathcal{H}^{\mathrm{DS}}=\bigoplus_{p \geq 1} \mathcal{H}_{p}^{\mathrm{DS}}
$$

where the $\mathcal{H}_{p}^{\mathrm{DS}}$ components consist of linear combinations of polylogarithms and Multiple Zeta Values (MZVs) of weight $p$. MZVs conjecturally form a graded subalgebra of their own,

$$
\mathcal{Z}=\bigoplus_{p \geq 0} \mathcal{Z}_{p}
$$

with $\mathcal{Z}_{0}=\mathbb{Q}$ and each component at weight $p$ has dimensionality $\left|\mathcal{Z}_{p}\right|:=\operatorname{dim}_{\mathbb{Q}} \mathcal{Z}_{p}$ with corresponding basis collected in the $p^{\text {th }}$ element of eq. (A.1).

In subsection 3.1, we first explain how to build a coproduct representation of $\mathcal{H}^{\mathrm{DS}}$, relying on the analytic properties worked out in section 2 . Then, in subsection 3.2 we promote this representation to explicit expressions in terms of MPLs. Finally, in subsection 3.3 carry out the $x \rightarrow 0$ series expansion of our functions, which will allow us to match them against predictions for the amplitude in the collinear limit, as we will discuss in the next section.

\subsection{Solving the integrability and extended Steinmann constraints}

Now let us turn to the construction of a particular basis $\left\{f_{j}^{(p)}\right\}_{j \in J_{p}}$ of the $\mathcal{H}_{p}^{\text {DS }}$ space of the DS functions of weight $p \geq 1$, where the elements of the basis are labeled by a list $J_{p}:=\left\{1,2, \ldots,\left|J_{p}\right|\right\}$. In this section we construct the $\mathcal{H}_{p}^{\mathrm{DS}}$ in the so-called coproduct form, then further refine it in section 2.4, and finally promote it to the full functional form in section 3.2. But before we do this, we first need to properly introduce the initial conditions and our notation for coproducts. 
The starting point of our recursive construction is the lowest weight space $\mathcal{H}_{1}^{\mathrm{DS}}$, which is two dimensional: $\operatorname{dim}_{\mathbb{Q}}\left(\mathcal{H}_{1}^{\mathrm{DS}}\right) \equiv\left|J_{1}\right|=2$, and is spanned by the following two logarithms:

$$
\begin{aligned}
& f_{1}^{(1)}:=G\left(\frac{1}{y} ; x\right)=\log (1-x y)=-\sum_{n \geq 1} \frac{1}{n}(x y)^{n}, \\
& f_{2}^{(1)}:=G(1 ; y)+G(0 ; x)=\log (x(1-y)) .
\end{aligned}
$$

Note, that around $x=0$ the first function $f_{1}^{(1)}$ has a well-defined Taylor expansion, while the second function $f_{2}^{(1)}$ is logarithmically divergent. In section 4 such power-and-log expansions of the elements of $\mathcal{H}^{\mathrm{DS}}$ will become our main tool for calculating amplitudes in the DS limit.

Next, as was outlined in section 2.1 , the $\{p-1,1\}$ coproduct component of a weight $p$ DS function $f_{i}^{(p)} \in \mathcal{H}_{p}^{\mathrm{DS}}$ can be expressed as a $\mathbb{Q}$-linear combination of certain $\otimes$-products with weight $(p-1)$ DS functions $f_{j}^{(p-1)} \in \mathcal{H}_{p-1}^{\mathrm{DS}}$ in their left entries and logarithms of the $\mathcal{A}$ alphabet elements in the right. As was first understood in [45] polylogarithmic functions are more economically expressed in terms of this coproduct component, and as advocated in [51], see also [54], it is more efficient to encode this component using a single object $c_{i j \alpha}^{(p)} \in \mathbb{Q}^{\left|J_{p}\right| \times\left|J_{p-1}\right| \times|\mathcal{A}|}$, which we refer to as the coproduct tensor, namely

$$
\Delta_{p-1,1}\left(f_{i}^{(p)}\right)=\sum_{j, \alpha} c_{i j \alpha}^{(p)} f_{j}^{(p-1)} \otimes \log (\alpha)
$$

where $i \in J_{p}$ and we have omitted the summation ranges $j \in J_{p-1}$ and $\alpha \in \mathcal{A}$ for simplicity. It follows from the coassociativity of the coproduct $\Delta$, that its $\{p-2,1,1\}$ component is then given by an inner product of two coproduct tensors at weight $p$ and $p-1$ :

$$
\Delta_{p-2,1,1}\left(f_{i}^{(p)}\right)=\sum_{\substack{j, k \\ \alpha, \beta}} c_{i j \alpha}^{(p)} c_{j k \beta}^{(p-1)} f_{k}^{(p-2)} \otimes \log (\beta) \otimes \log (\alpha) .
$$

Now we are ready to discuss the recursive step, i.e. the construction of the $\mathcal{H}_{p}^{\mathrm{DS}}$ out of the already known $\mathcal{H}_{p-1}^{\mathrm{DS}}$ space at previous weight. Following the coproduct bootstrap method, we formulate a set of linear constraints on the coproducts, whose nullspace defines the $\mathcal{H}_{p}^{\mathrm{DS}}$ space. To do that, we start with a tensor product $\Delta_{p-2,1}\left(\mathcal{H}_{p-1}^{\mathrm{DS}}\right) \otimes \mathcal{A}$ of the $\{p-2,1\}$ coproduct component of the whole $\mathcal{H}_{p-1}^{\mathrm{DS}}$ space, encoded in the $c_{j k \beta}^{(p-1)}$ tensor, and another copy of the $\mathcal{A}$ alphabet. Using the natural inclusion $\Delta_{p-2,1}\left(\mathcal{H}_{p-1}^{\mathrm{DS}}\right) \otimes \mathcal{A} \subset \mathcal{H}_{p-2}^{\mathrm{DS}} \otimes \mathcal{A} \otimes \mathcal{A}$, we impose a set $\mathcal{S}:=\{1, \ldots,|\mathcal{S}|\}$ of homogeneous linear conditions on the last two entries of the coproduct via a map $\mathcal{I}: \mathcal{A} \otimes \mathcal{A} \rightarrow \mathbb{Q}^{|\mathcal{S}|}$ that acts on the tensor space as follows:

$$
\mathcal{H}_{p-1}^{\mathrm{DS}} \otimes \mathcal{A} \stackrel{\Delta_{p-2,1} \otimes \mathrm{id}}{\longrightarrow} \Delta_{p-2,1}\left(\mathcal{H}_{p-1}^{\mathrm{DS}}\right) \otimes \mathcal{A} \stackrel{\mathrm{id} \otimes \mathcal{I}}{\longrightarrow} \mathcal{H}_{p-2}^{\mathrm{DS}} \otimes \mathbb{Q}^{|\mathcal{S}|}
$$

and look for its kernel. In our implementation $\mathcal{I}$ contains the integrability as well as the extended Steinmann conditions shown in eqs. (2.31) and (2.32), so that $|\mathcal{S}|=8$ in our case. In practice, the map $\mathcal{I}$ is represented as a tensor $\mathcal{I}_{\alpha \beta s} \in \mathbb{Q}^{|\mathcal{A}| \times|\mathcal{A}| \times|\mathcal{S}|}$, whose explicit values are stated in eq. (B.2). Contraction of the $\mathcal{I}_{\alpha \beta s}$ tensor together with the coproduct tensor 


\begin{tabular}{|c|cccccc|}
\hline weight & 2 & 3 & 4 & 5 & 6 & 7 \\
number & 5 & 12 & 26 & 56 & 116 & 236 \\
non-zero & 12 & 31 & 96 & 263 & 901 & $2.6 \times 10^{3}$ \\
density & 0.24 & 0.10 & 0.06 & 0.04 & 0.03 & 0.019 \\
max & 1 & 2 & 2 & 20 & 40 & 560 \\
\hline weight & 8 & 9 & 10 & 11 & 12 & 13 \\
number & 474 & 943 & 1867 & 3686 & 7270 & 14295 \\
non-zero & $9.9 \times 10^{3}$ & $39 \times 10^{3}$ & $.2 \times 10^{6}$ & $.8 \times 10^{6}$ & $3.6 \times 10^{6}$ & $15 \times 10^{6}$ \\
density & 0.018 & 0.018 & 0.024 & 0.025 & 0.026 & 0.029 \\
max & 1120 & $5.6 \times 10^{4}$ & $6.0 \times 10^{8}$ & $1.9 \times 10^{12}$ & $5.7 \times 10^{17}$ & $2.0 \times 10^{25}$ \\
\hline
\end{tabular}

Table 2. Features of the $\mathcal{H}_{p}^{\mathrm{DS}}$ basis we have constructed, in its coproduct tensor representation. The first row represents the weight $p$, the second row gives the dimension of the space (including MZVs), the third, fourth and fifth rows show the amount of non-zero entries, densities and maximum entry values of the coproduct tensors, respectively.

$c_{j k \beta}^{(p-1)}$ gives a realization of the $M:=[\mathrm{id} \otimes \mathcal{I}] \circ\left[\Delta_{p-2,1} \otimes \mathrm{id}\right]$ map from eq. (3.7), whose tensorial representation (after some transpositions) can be easily spelled out:

$$
M_{k s j \alpha}:=\sum_{\beta} c_{j k \beta}^{(p-1)} \mathcal{I}_{\alpha \beta s} .
$$

A more explicit derivation of this definition is given in appendix B. This map $M$ encodes the basic linear constraints needed for determining the $\mathcal{H}_{p}^{\mathrm{DS}}$ space.

Now, let $\tilde{c}_{\tilde{i}(j \alpha)}^{(p)} \in \mathbb{Q}^{\left|\tilde{J}_{p}\right| \times\left(\left|J_{p-1}\right| \cdot|\mathcal{A}|\right)}$ denote a basis of the $\operatorname{Ker}\left(M_{(k s)(j \alpha)}\right)$ nullspace:

$$
\sum_{(j \alpha)} M_{(k s)(j \alpha)} \tilde{c}_{\tilde{i}(j \alpha)}^{(p)}=0, \quad \text { for each } k \in J_{p-1}, s \in \mathcal{S}, \tilde{i} \in \tilde{J}_{p},
$$

where the list $\tilde{J}_{p}:=\left\{1, \ldots,\left|\tilde{J}_{p}\right|\right\}$, for the moment, labels the basis elements, while the brackets $(j \alpha)$ and $(k s)$ denote vectorization of the corresponding tensor indices, which label bases of the leftmost and the rightmost spaces in eq. (3.7) respectively. To further reduce the nullspace $\operatorname{Ker}\left(M_{(\alpha j)(s k)}\right)$ and obtain the entire $\mathcal{H}_{p}^{\mathrm{DS}}$ space we exploit the additional analyticity constraints shown in eq. (2.38). Each of these 3 branch cut conditions always evaluate to just MZVs, hence they produce $3 \times\left|\mathcal{Z}_{p-1}\right|$ additional linear equations at weight $p$. Resolving these conditions further reduces the list of basis elements $\tilde{J}_{p} \rightarrow J_{p}$, and leaves us with the final form of the $c_{i j \alpha}^{(p)}$ coproduct tensors. Their properties with respect to the weight $p$ are summarized in table 2 . Note, in particular, that the produced coproduct tensors tend to be very sparse and their densities never exceed a few percent. We present the coproduct tensors for weight $p \leq 13$ in the ancillary file coproducts-w2-13.m.

In practice, the natural $\operatorname{GL}\left(\left|\tilde{J}_{p}\right|\right)$ freedom of choosing a particular version of the coproduct tensors (prior to imposing the branch cut conditions) $\tilde{c}_{\tilde{i}(j \alpha)}^{(p)}$ can be used to improve the efficiency of the computer implementation. There are, of course, many possible 


\begin{tabular}{|c|cccc|}
\hline weight & A & B & C & D \\
\hline 2 & 1 & 1 & 1 & 1 \\
3 & 2 & 2 & 1 & 1 \\
4 & 2 & 2 & 2 & 2 \\
5 & 20 & 20 & 10 & 7 \\
6 & 40 & 20 & 60 & 105 \\
7 & 560 & 420 & 2100 & 18366 \\
8 & 1120 & 14850 & 5950 & 227673167 \\
\hline
\end{tabular}

Table 3. The sizes of maximal entries of the coproduct tensors $c_{i j \alpha}^{(p)}$ at weight $p$ in the four computational setups that we used. The version A, our best choice so far, reorders the rows of the tensors and uses the LLL reduction; the version $\mathrm{B}$ in addition to that also rescales the rows of the tensors in order to make them integer-valued and only then applies the LLL; the version C only uses the LLL without any reordering of the rows; version D does not modify the tensors in any way and serves as a baseline for numeric optimizations.

metrics for optimization: we can look for the most sparse tensors in the output, the fastest overall execution time, the lowest values of the $\|\cdot\|_{\infty}$ norm, or some heuristic mix of those. The main computational challenge is the Gaussian elimination in the field of rationals $\mathbb{Q}$ required for the nullspace determination. As the dimensions of the equation matrix $M_{(k s)(j \alpha)}$ grow exponentially with increasing weight $p$, so do the denominators and numerators in its entries. A very special care is therefore needed in order to perform the Gaussian elimination at higher weights. Building on the ideas of [48], we tested several possible optimization strategies, the most promising of which are presented in table 3 . Our best method of computation consists of a reordering of vectors in the nullspace basis (at low weights obtained via MATHEMATICA's Nullspace command) with respect to the number of non-zero entries in them (from small to big), and a subsequent application of the Lenstra-Lenstra-Lovász (LLL) reduction via MATHEMATICA's LatticeReduce command. The purpose of the reordering is to simplify the equation matrix $M_{(k s)(j \alpha)}$ at the next weight by bringing it closer to the row echelon form, which helps to avoid the excessive fill-in of zero elements during the following Gaussian elimination. The LLL reduction significantly decreases the sizes of the numerators and denominators in the entries of the produced coproduct tensors $c_{j k \beta}^{(p)}$, as reflected in table 3 . At higher weights $p \geq 11$ we also made use of the Spasm library [83] for performing the row reduction over finite fields and subsequent rational reconstruction.

\subsection{Promoting coproducts to functions}

Now everything is ready for construction of the full function space $\mathcal{H}^{\mathrm{DS}}$ that is used to determine the MHV and NMHV six-gluon amplitudes in the DS limit. We provide two equivalent representations of the elements of $\mathcal{H}^{\mathrm{DS}}$ : one in terms of MPLs and the other one in terms of power-and-log series expansions. The latter is less computationally demanding and instrumental for the Wilson loop OPE resummation as explained in section 4.2. 


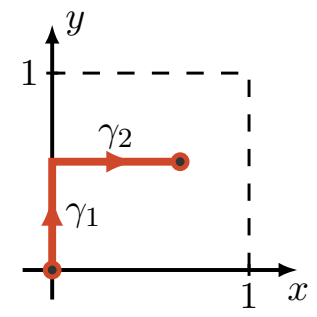

Figure 2. Integration path $\gamma:=\gamma_{1} \gamma_{2}$ connecting the base point of integration $\{0,0\}$ and a general kinematical point $\{x, y\}$ inside the unit square.

\begin{tabular}{|c|ccccc|}
\hline alphabet $\mathcal{A}$ & $x$ & $y$ & $1-x$ & $1-y$ & $1-x y$ \\
\hline$\gamma_{1}$ & 0 & $d \log (y)$ & 0 & $d \log (y-1)$ & 0 \\
$\gamma_{2}$ & $d \log (x)$ & 0 & $d \log (x-1)$ & 0 & $d \log \left(x-\frac{1}{y}\right)$ \\
\hline
\end{tabular}

Table 4. Pull-backs of the $d$ log-forms along the two components of the integration path shown in figure 2. These $d \log$-forms act as the integration kernels in eqs. (3.11), (3.12) and (3.13). Note, that along $\gamma_{1}$ the integration variable is $y$, and along $\gamma_{2}$ the integration variable is $x$, while $y$ remains constant.

Our procedure essentially boils down to iterative integration of the differentials (2.4) defined by the coproducts from eq. (3.5) along a fixed path $\gamma$ shown in figure 2. The path $\gamma:=\gamma_{1} \gamma_{2}$ connects the base point of integration $\{x, y\}=\{0,0\}$, at which we set the integration constants to 0 , with a general kinematical point $\{x, y\}$ inside the unit square $0<x, y<1$. Our choice of the integration path $\gamma$ dictates a special representation of the DS functions $f_{j}^{(p)} \in \mathcal{H}_{p}^{\mathrm{DS}}$ in terms of MPLs, see for example [84] or [85],

$$
f_{j}^{(p)}=\sum_{\vec{X}, \vec{Y}} g_{j \vec{X} \vec{Y}} G(\vec{X} ; x) G(\vec{Y} ; y), \quad g_{j \vec{X} \vec{Y}} \in \mathcal{Z}^{\left|J_{p}\right| \times 3^{p} \times 2^{p}}, \quad\left|g_{j \vec{X} \vec{Y}}\right|+|\vec{X}|+|\vec{Y}|=p
$$

where the lists (which are also referred to as 'words') of arguments $\vec{Y}$ and $\vec{X}$ are drawn from the $\vec{Y} \in\{0,1\}^{\bullet}$ and $\vec{X} \in\left\{0,1, \frac{1}{y}\right\}^{\bullet}$ sets. Here by $\Sigma^{\bullet}$ we mean the set of all words made out of some finite set $\Sigma$, in other words it is a disjoint union $\Sigma^{\bullet}:=\epsilon \dot{\cup} \Sigma \dot{U} \Sigma^{2} \dot{U} \ldots$, where $\epsilon$ is an empty word, while any other word $\vec{X}$ of length $|\vec{X}|$ lies in the $|\vec{X}|^{\text {th }}$ component: $\vec{X} \in \Sigma^{|\vec{X}|}$. The lengths of these two lists and the transcendental weight of the MZV-valued coefficients, denoted as $|\vec{X}|,|\vec{Y}|$, and $\left|g_{j \vec{X} \vec{Y}}\right|$ respectively, add up to the transcendental weight $p$ of the function for each term in the sum.

The integral along $\gamma$ of the $\{p-1,1\}$ coproduct component (3.5) or, equivalently, of the differential of a given DS function splits into two terms corresponding to the two parts of the integration path. The pull-backs of the $d \log$-forms shown in table 4 make sure that both of these integrations preserve the general form of the (3.10) representation: the integral along the $\gamma_{1}$ only modifies the $g_{j \epsilon \vec{Y}} G(\vec{Y} ; y)$ terms in that sum, while the $\gamma_{2}$ integration changes only the $G(\vec{X} ; x)$ factor. To quantitatively describe the second integral along the $\gamma_{2}$ path 
we introduce the following three linear operators:

$$
\begin{aligned}
& \mathcal{O}_{1}(G(\vec{X} ; x)):=\int_{0}^{x} G(\vec{X} ; t) d \log (t)=G(0, \vec{X} ; x), \\
& \mathcal{O}_{2}(G(\vec{X} ; x)):=\int_{0}^{x} G(\vec{X} ; t) d \log (t-1)=G(1, \vec{X} ; x), \\
& \mathcal{O}_{3}(G(\vec{X} ; x)):=\int_{0}^{x} G(\vec{X} ; t) d \log \left(t-\frac{1}{y}\right)=G\left(\frac{1}{y}, \vec{X} ; x\right) .
\end{aligned}
$$

It is evident that these operators just prepend the corresponding letter to the list of arguments. Later in eqs. (3.19)-(3.21) we will show how the same integral operators act on the series expansions of MPLs, which turn out to be more useful objects in practice.

In this manner, we have succeeded in promoting the coproduct representation of our DS functions to explicit expressions in terms of MPLs through weight 12. Due to size restrictions, in the ancillary file ds-w1-10.m we provide these MPL representations through weight 10 and list the weight $p \leq 3$ functions explicitly below. ${ }^{7}$ In terms of the following compact notation,

$$
l_{x}:=\log (x), \quad \mathcal{G}_{\vec{X}}:=G(\vec{X} ; x) .
$$

which we will adopt from this point on, the weight $p=1$ functions of eqs. (3.3) and (3.4) read

$$
f_{1}^{(1)}=l_{1-x y}, f_{2}^{(1)}=l_{x(1-y)} .
$$

Then, there are five functions at weight $p=2$,

$$
\begin{aligned}
& f_{1}^{(2)}=\mathcal{G}_{0 \check{y}}, f_{2}^{(2)}=\mathcal{G}_{0 \check{y}}-\mathcal{G}_{1 \check{y}}-\mathcal{G}_{01}+l_{1-x} l_{x(1-y)}, \\
& f_{3}^{(2)}=\frac{1}{2} l_{x(1-y)}^{2}, f_{4}^{(2)}=l_{x(1-y)} l_{1-x y}-l_{1-x y}^{2}, f_{5}^{(2)}=\zeta_{2},
\end{aligned}
$$

and 12 functions at $p=3$,

$$
\begin{aligned}
f_{1}^{(3)} & =\mathcal{G}_{\check{y} 0 \check{y}}, f_{2}^{(3)}=\zeta_{2} l_{1-x y}, f_{3}^{(3)}=\zeta_{2} l_{x(1-y)}, f_{4}^{(3)}=\mathcal{G}_{00 \check{y}}, \\
f_{5}^{(3)} & =\mathcal{G}_{01} l_{x(1-y)}+\mathcal{G}_{00 \check{y}}-\mathcal{G}_{01 \check{y}}-2 \mathcal{G}_{001}, f_{6}^{(3)}=\frac{1}{6} l_{x(1-y)}^{3}, \\
f_{7}^{(3)} & =\frac{1}{2} l_{1-x y} l_{x(1-y)}^{2}+l_{x(1-y)}\left[-\mathcal{G}_{0 \check{y}}-\mathcal{G}_{\check{y} 1}\right]+\mathcal{G}_{00 \check{y}}+\mathcal{G}_{0 \check{y} 1}+\mathcal{G}_{\check{y} 01}-\mathcal{G}_{\breve{y} 0 \check{y}}+\mathcal{G}_{\check{y} 1 \check{y}}, \\
f_{8}^{(3)} & =\mathcal{G}_{0 \check{y}} l_{x(1-y)}-2 \mathcal{G}_{0 \check{y} \check{y}}, \\
f_{9}^{(3)} & =\frac{1}{2} l_{1-x} l_{x(1-y)}^{2}+l_{x(1-y)}\left[-\mathcal{G}_{01}-\frac{1}{2} l_{1-x}^{2}\right]-\mathcal{G}_{10 \check{y}}+\mathcal{G}_{11 \check{y}}+\mathcal{G}_{001}+\mathcal{G}_{011}+\mathcal{G}_{101}+\zeta_{2} l_{1-x}, \\
f_{10}^{(3)} & =l_{x(1-y)}\left[\mathcal{G}_{0 \check{y}}-\mathcal{G}_{1 \check{y}}\right]-\mathcal{G}_{00 \check{y}}+\mathcal{G}_{01 \check{y}}-2 \mathcal{G}_{0 \check{y} \check{y}}+2 \mathcal{G}_{1 \check{y} \check{y}}, \\
f_{11}^{(3)} & =l_{x(1-y)}\left[-\mathcal{G}_{1 \check{y}}-\mathcal{G}_{\check{y} 1}+l_{1-x y}^{2}\right]+\mathcal{G}_{01 \check{y}}+\mathcal{G}_{0 \check{y} 1}-2 \mathcal{G}_{0 \check{y} \check{y}}+2 \mathcal{G}_{1 \check{y} \check{y}}+\mathcal{G}_{\check{y} 01}-\mathcal{G}_{\check{y} 0 \check{y}}+\mathcal{G}_{\check{y} 1 \check{y}}-\frac{2}{3} l_{1-x y}^{3}, \\
f_{12}^{(3)} & =\zeta_{3} .
\end{aligned}
$$

As was anticipated at the end of section 2.2, we see the low-weight MZV constants $\zeta_{2}$ and $\zeta_{3}$ are independent elements of the $\mathcal{H}^{\mathrm{DS}}$ space, the same holds for other MZVs from eq. (A.1) at higher weights.

\footnotetext{
${ }^{7}$ Readers interested in the $p=11,12$ expressions are welcome to contact the authors.
} 
Before concluding this subsection, let us also make a technical remark regarding the efficient representation of MPLs for computer algebra manipulations [86]: it proves useful to store the $\vec{Y}$ lists of arguments of MPLs from eq. (3.10) as base 2 (or binary) numbers, and encode the $\vec{X}$ lists as base 3 (or ternary) numbers via a $\frac{1}{y} \rightarrow 2$ replacement, e.g. $G\left(1,0, \frac{1}{y} ; x\right) \mapsto G\left(102_{3} ; x\right)$. It allows us to represent the action of the $\left\{\mathcal{O}_{1}, \mathcal{O}_{2}, \mathcal{O}_{3}\right\}$ integration operators arithmetically as addition of certain (weight-dependent) numbers, e.g. $\mathcal{O}_{3}\left(G\left(1,0, \frac{1}{y} ; x\right)\right)=G\left(\frac{1}{y}, 1,0, \frac{1}{y} ; x\right) \mapsto G\left(102_{3}+2000_{3} ; x\right)$. Also note that as a consequence of the branch cut condition of eq. (2.38), $f^{y}=0$ as $y \rightarrow 0$, the first integral along the $\gamma_{1}$ effectively only increases the powers of $l_{1-y}:=\log (1-y)$, since the $\vec{Y}$ lists in eq. (3.10) are forced to be free of any zeros inside of them. Therefore we only need to keep track of its overall length $|\vec{Y}|$. We have found that these tweaks greatly reduce the memory and storage usage of our computer implementation.

\subsection{Promoting coproducts to expansions}

For every function inside our $\mathcal{H}^{\mathrm{DS}}$ space, apart from its MPL representation $f$ we also construct its $x \rightarrow 0$ expansion, which we shall denote as $\check{f}$ in order to avoid confusion (note that we have also dropped the weight index to avoid clutter). In the next section we will use these expansions $\check{f}$ to resum the Wilson loop OPE state sum and obtain predictions for the NMHV six gluon amplitude at high loop orders, so before that we need to discuss the relevant features of our construction.

In fact, direct integration of the $x \rightarrow 0$ expansions $\check{f}$ turns out to be easier than expanding the MPL representations we obtained in the previous subsection, especially at higher weights $p \geq 10$. This way at each step of the algorithm we only have to deal with $x \rightarrow 0$ power-and-log expansions of MPLs, which are directly applied to fix the coefficient of the ansatz in the NMHV amplitude bootstrap problem. We will consider the $x \rightarrow 0$ expansions $\check{f}$ of the following mixed form:

$$
\check{f}=\sum_{k=0}^{N} \sum_{l=0}^{k-1} \sum_{m, n} q_{k l m n} x^{k} y^{l}(\log (x))^{m}(\log (1-y))^{n}+\sum_{\tilde{X}} g_{\tilde{X}} G(\tilde{X} ; x)
$$

where the list of arguments of MPLs $\tilde{X}$ is drawn from a reduced set $\tilde{X} \in\left\{0, \frac{1}{y}\right\}^{\bullet}$, and the coefficients $q_{k l m n} \in \mathcal{Z}^{N \times(N-1) \times p \times p}$ and $g_{\tilde{X}} \in \mathcal{Z}^{2^{p}}$ are MZV-valued, so that the overall weight of the r.h.s. in eq. (3.18) does not exceed $p$, while $\left|g_{\tilde{X}}\right|+|\tilde{X}|=p$. The summation range $0 \leq l<k \leq N$ in the first sum of eq. (3.18) indicates that the MPLs that contribute to it have at least one " 1 " among their arguments. In contrast, MPLs from the second sum of eq. (3.18), when expanded, only contain equal powers of both $x$ and $y$ (apart from simple logarithms). Therefore, we refer to the first sum in eq. (3.18) as the "non-diagonal part" of the expansion and denote the corresponding linear space of monomials by $\mathcal{N}$, while the second sum will be called the "diagonal part", whose linear space of MPLs (or $x \rightarrow 0$ expansions) is denoted by $\mathcal{D}$. Hence an expansion $\check{f}$ belongs to a direct sum of these two parts: $\check{f}=\mathcal{N} \oplus \mathcal{D}$ and so does the NMHV amplitude that we are after.

Alongside the $\{p-1,1\}$ coproduct components discussed in section 3.1, our main computational tool are the integration operators introduced in eqs. eq. (3.11)-(3.13). Their 


\begin{tabular}{|c|cc|}
\hline & $\mathcal{N}$ & $\mathcal{D}$ \\
\hline $\mathcal{O}_{1}$ & $\mathcal{N}$ & $\mathcal{D}$ \\
$\mathcal{O}_{2}^{\prime}$ & $\mathcal{N}$ & $\mathcal{N}$ \\
$\mathcal{O}_{3}^{\prime}$ & $\mathcal{N}$ & $\mathcal{D}$ \\
\hline
\end{tabular}

Table 5. Action of the integration operators $\left\{\mathcal{O}_{1}, \mathcal{O}_{2}^{\prime}, \mathcal{O}_{3}^{\prime}\right\}$ on the non-diagonal $\mathcal{N}$ and diagonal $\mathcal{D}$ parts of the expansions. None of the operators maps the non-diagonal part $\mathcal{N}$ into the diagonal $\mathcal{D}$, and the $\mathcal{O}_{1}$ and $\mathcal{O}_{3}^{\prime}$ operators even preserve $\mathcal{D}$. These properties allow us to keep the diagonal $\mathcal{D}$ part of the expansion in eq. (3.18) in the full MPL form and only expand the non-diagonal $\mathcal{N}$ part.

action on the non-diagonal $\mathcal{N}$ part of the expansions $\check{f}$ is given by the following formulas:

$$
\begin{aligned}
\left.\mathcal{O}_{1}(\check{f})\right|_{\mathcal{N}} & :=\sum_{k, l, m, n} q_{k l m n} x^{k} y^{l}(\log (1-y))^{n} \sum_{i=0}^{m}(-1)^{m-i} \frac{m !}{i !} k^{-(m-i+1)}(\log (x))^{i} \\
\left.\mathcal{O}_{2}^{\prime}(\check{f})\right|_{\mathcal{N}} & :=-\sum_{k, l, m, n} q_{k l m n} y^{l}(\log (x))^{m}(\log (1-y))^{n} \sum_{i=k+1}^{N} x^{i} \\
\left.\mathcal{O}_{3}^{\prime}(\check{f})\right|_{\mathcal{N}} & :=-\sum_{k, l, m, n} q_{k l m n} y^{l-k}(\log (x))^{m}(\log (1-y))^{n} \sum_{i=k+1}^{N}(x y)^{i}
\end{aligned}
$$

where the partial operators $\left\{\mathcal{O}_{2}^{\prime}, \mathcal{O}_{3}^{\prime}\right\}$ are related to the full integration operators $\left\{\mathcal{O}_{2}, \mathcal{O}_{3}\right\}$ as $\mathcal{O}_{2}=\mathcal{O}_{1} \circ \mathcal{O}_{2}^{\prime}$ and $\mathcal{O}_{3}=\mathcal{O}_{1} \circ \mathcal{O}_{3}^{\prime}$. Note, that the rule (3.19) needs an additional definition in case of a pure $\log (x)$ monomial:

$$
\mathcal{O}_{1}\left((\log (x))^{m}\right):=\frac{1}{m+1}(\log (x))^{m+1},
$$

in accordance with eq. (2.8).

The action of $\left\{\mathcal{O}_{1}, \mathcal{O}_{2}^{\prime}, \mathcal{O}_{3}^{\prime}\right\}$ is summarized in table 5 in terms of the non-diagonal and diagonal parts $\mathcal{N}$ and $\mathcal{D}$. A crucial observation about these operators is that they do not increase the power of $y^{l}$ independently from $x^{k}$ or, in other words, none of them act as $\mathcal{N} \rightarrow \mathcal{D}$, which is, of course, just a consequence of the eqs. (3.11)-(3.13). This feature allows us to work with the diagonal part $\mathcal{D}$ of any expansion $\check{f}$ separately keeping it in the full unexpanded MPL form.

Finally, let us note an important technical detail of this approach: the maximal expansion order $N$ needed for the high-weight functions has to be set in advance for the weight $p=1$ functions, and cannot be altered in between. We define the optimal expansion order $N(p)=N_{\text {opt }}(p)$ to be the minimal value of $N$, for which the expansions of the weight $p$ functions we obtain in this way, are linearly independent. Our empirical findings of the value of $N_{\text {opt }}(p)$ are summarized in table 6 . We emphasize that had we chosen to also expand the diagonal part $\mathcal{D}$, the optimal expansion order would drastically increase. The computational gain obtained thus justifies our hybrid strategy of only expanding the non-diagonal $\mathcal{N}$ part in eq. (3.18). 


\begin{tabular}{|c|ccccccc|}
\hline weight $p$ & $\leq 6$ & 7 & 8 & 9 & 10 & 11 & 12 \\
order $N_{\text {opt }}$ & $\leq 4$ & 5 & 7 & 9 & 13 & 18 & 25 \\
\hline
\end{tabular}

Table 6. Optimal expansion order $N_{\text {opt }}$, corresponding to the minimal value of $N$ in eq. (3.18), such that the series expansions $\check{f}$ of all DS functions at a given weight $p$ become linearly independent.

\section{The Wilson loop OPE and the collinear limit}

In the previous sections, we essentially described how to construct an ansatz for the six-gluon amplitude in the DS limit. Here, we will show how to find a unique solution for this ansatz, by exploiting independent information for the amplitude in the collinear boundary of the DS limit, furnished by the Wilson Loop OPE approach [10-19].

While our space of functions contains both the MHV and NMHV amplitudes, in what follows we will be focusing on the latter, which in the literature is known to lower loop order [53]. After reviewing another amplitude normalization and set of kinematic variables that will be convenient for our purposes, in subsection 4.1 we analyze the behavior of the rational, $R$-invariant part of the NMHV amplitude in the two parity-conjugate DS limits. Choosing one of the two limits, and the so-called (1111) rational component of the amplitude, subsection 4.2 then discusses in detail how to obtain predictions from the Wilson loop OPE, and match them against our ansatz. The reader interested in the final result, may jump to section 5 .

\subsection{Wilson loop normalization, variables and $R$-invariants in the DS limit}

In this section, we start by reviewing some useful notation on the NMHV Wilson loop, mostly relying on ref. [53]. Expert readers may jump straight to eq. (4.6), where we proceed to specialize this notation to the DS limit. In order to describe the Wilson loop dual to the amplitude, it will be convenient to change the BDS-like normalization (2.1), redistributing a known factor between the finite and infrared-divergent part of the latter. That is, in this section we will be considering the NMHV framed Wilson loop, $W$, which is related to E by

$$
\frac{\mathrm{E}}{W}=\exp \left(-\frac{1}{4} \Gamma_{\operatorname{cusp}} \mathcal{X}\right)
$$

In the rightmost factor in this equation $\frac{1}{4} \Gamma_{\text {cusp }}=g^{2}-2 \zeta_{2} g^{4}+\mathcal{O}\left(g^{6}\right)$ is the cusp anomalous dimension, a quantity known to all loops [87], and

$$
\begin{aligned}
\mathcal{X}:= & X-\mathcal{E}^{(1)} \\
X= & -\operatorname{Li}_{2}(1-u)-\operatorname{Li}_{2}(1-v)+\operatorname{Li}_{2}(w) \\
& +\log (1-w)^{2}-\log (1-w) \log \left(\frac{v}{u}\right)-\log (u) \log (v)+\frac{\pi^{2}}{6}, \\
\mathcal{E}^{(1)}= & \operatorname{Li}_{2}\left(1-\frac{1}{u}\right)+\operatorname{Li}_{2}\left(1-\frac{1}{v}\right)+\operatorname{Li}_{2}\left(1-\frac{1}{w}\right) .
\end{aligned}
$$

As we mentioned in section 2 , at each loop order $L, \mathcal{E}^{(L)}$ is believed to be a pure transcendental function, and particularly in the DS limit it should lie in the $\mathcal{H}^{\mathrm{DS}}$ space. On the other hand, as shown in eq. (2.2) $\mathrm{E}$ is a combination of pure functions and rational 
$R$-invariants. The latter are polynomials in the (dual) Graßmann variables $\eta_{i}^{A}$ encoding the supersymmetry of the theory, where $A=1, \ldots, 4$ is an R-symmetry index, and $i=1, \ldots, 6$ is related to the cyclic ordering of scattered particles. In this work we will particularly focus on their (1111) component, which is proportional to the $\eta_{1}^{1} \eta_{1}^{2} \eta_{1}^{3} \eta_{1}^{4}$ monomial in the Graßmann variables inside of the $R$-invariants, first studied from the point of view of the Wilson loop OPE in [11].

Following the latter reference, we will also switch to another choice of kinematic variables that is convenient for describing both the collinear and DS limits of the hexagonal Wilson loop. It consists of a triple $\{S, F, T\}$, whose relation to the $\{u, v, w\}$ cross-ratios reads:

$$
u=\frac{F}{F+F S^{2}+S T+F^{2} S T+F T^{2}}, \quad v=\frac{S^{2}}{T^{2}} u w, \quad w=\frac{T^{2}}{1+T^{2}} .
$$

In these variables, for the NMHV (1111) component of interest the $R$-invariants reduce to [53]

$$
\begin{aligned}
& (1) \rightarrow 0, \\
& (2) \rightarrow \frac{F^{3} T}{(S+F T)\left(F+S T+F T^{2}\right)\left(F+F S^{2}+S T+F^{2} S T+F T^{2}\right)}, \\
& (3) \rightarrow \frac{1}{\left(1+T^{2}\right)\left(1+F S T+T^{2}\right)}, \\
& (4) \rightarrow \frac{S}{S+F T}, \\
& (5) \rightarrow \frac{T(F S+T)^{3}}{F\left(1+F S T+T^{2}\right)\left(F+F S^{2}+S T+F^{2} S T+F T^{2}\right)}, \\
& (6) \rightarrow \frac{T^{4}}{F\left(1+T^{2}\right)\left(F+S T+F T^{2}\right)} .
\end{aligned}
$$

Now let's see what are the simplifications to the above formulas that follow from the double scaling kinematics. As was shown in section 2.2, there are really two DS limits, which in the OPE variables (4.4) correspond to:

$$
\begin{array}{lll}
\text { double scaling limit 1 (DS1): } & \left\{T, F^{-1}\right\} \rightarrow 0, & T F \text { fixed, } \\
\text { double scaling limit 2(DS2): } & \{T, F\} \rightarrow 0, & T F^{-1} \text { fixed. }
\end{array}
$$

They are related by the parity transformation, in these variables translates to a simple $F \rightarrow F^{-1}$ replacement. In these limits, we may also relate the $\{x, y\}$ variables of eq. (2.26) with respect to the surviving OPE variables as $\left.\{x, y\}\right|_{\mathrm{DS} 1}=\left\{-\frac{T F}{S}, 1+\frac{1}{T F S}\right\}$ and

$$
\left.\{x, y\}\right|_{\mathrm{DS} 2}=\left\{-\frac{T}{S F}, 1+\frac{F}{T S}\right\} .
$$

From now on will thus switch back to our familiar $\{x, y\}$ variables.

Also note that the cross-ratios eq. (4.4) are parity-even functions, so they reduce in both versions of the DS limit to the same values, $\{u, v, w\} \rightarrow\left\{\frac{x(1-y)}{1-x y}, \frac{1}{1-x y}, 0\right\}$. Therefore, 
the function $\mathcal{X}$ responsible for the BDS-like normalization and defined in eq. (4.2) in both of the DS limits reduces to

$$
\mathcal{X} \underset{\mathrm{DS}}{\longrightarrow} \frac{\pi^{2}}{3}+\frac{1}{2} \log (x(1-y))+\frac{1}{2} \log (w)^{2} .
$$

On the other hand, the (1111) components of the $R$-invariants in the two versions of the double scaling limit differ significantly. Using the definitions from eqs. (4.5) and the limits from eqs. (4.6) and (4.7) we see that

$$
\{(1), \ldots,(6)\} \rightarrow \begin{cases}\left\{0,-\frac{x^{2}(1-y)}{(1-x)(1-x y)},-\frac{1-y}{y}, \frac{1}{1-x}, \frac{1}{y(1-x y)}, 0\right\} & \text { for DS1 } \\ \{0,0,1,1,0,0\} & \text { for DS2 }\end{cases}
$$

Very interestingly, the DS2 limit completely trivializes the $R$-invariants and makes the ratio function $\mathrm{E}^{(1111)}$ pure (meaning it becomes a $\mathbb{Q}$-linear sum of MPLs). From this point on, we will focus on the DS2 limit as it greatly facilitates the search for the NMHV six-particle amplitude within the DS function space $\mathcal{H}^{\mathrm{DS}}$. Specifically, it allows us to use the same mixed form of eq. (3.18) for the collinear limit expansion of the entire amplitude, without having to take additional contributions from the rational factors into account.

\subsection{NMHV Wilson loop OPE}

Let us now proceed to discuss in detail how to determine the (1111) component of the six-particle NMHV amplitude in the DS2 limit with the help of the Wilson loop OPE. We start by briefly reviewing the latter approach, and especially the predictions it provides for the weak-coupling expansion of the amplitude in integral form, mostly relying on [13, 27]. Readers familiar with this material may skip to eq. (4.28), where we proceed to trade them with infinite sum representations relying on Cauchy's residue theorem. Finally, we match them against the series representation of our ansatz, carried out in subsection 3.3.

The Wilson loop OPE provides a non-perturbative description of the amplitude as a state sum expansion around the collinear limit. The DS and collinear limits are closely related as can be seen from eqs. (4.6) and (4.7). The DS limit benefits from having only gluonic states with positive (for DS1) or negative (for DS2) helicities contributing to it. In the DS2 limit we will be focusing on, discussed in the previous subsection, the (1111) component of the framed NMHV Wilson loop $W$ may be written as

$$
W \underset{\mathrm{DS2}}{\longrightarrow} \sum_{N_{-} \geq 0} W_{N_{-}}=\sum_{N_{-} \geq 0} \sum_{L \geq 0} g^{2 L} W_{N_{-}}^{(L)},
$$

where $W_{N_{-}}$are contributions from multi-particle excitations consisting of $N_{-}$negative helicity gluon bound states, further organized into $L$-loop contributions to their weakcoupling expansion. As we'll see below, $W_{N_{-}}$starts contributing at $L=N_{-}^{2}+N_{-}$loops, and so for our purposes it will be sufficient to restrict to $N \leq 2$, which provides an accurate description up to and including $L=11$ loops.

The one gluon bound state particle contribution $W_{1_{-}}$is given by

$$
W_{1_{-}}=\sum_{l_{1} \geq 1}\left(T F^{-1}\right)^{l_{1}} \int \frac{\mathrm{d} u_{1}}{2 \pi} T^{\gamma_{l_{1}}\left(u_{1}\right)} S^{\mathrm{i} p_{l_{1}}\left(u_{1}\right)} \times \mu_{l_{1}}\left(u_{1}\right) h_{-l_{1}}\left(u_{1}\right),
$$


whereas the superposition of two gluon bound states $W_{2_{-}}$by

$$
\begin{aligned}
W_{2_{-}}=\sum_{l_{1} \geq l_{2} \geq 1} \frac{1}{1+\delta_{l_{1}, l_{2}}}\left(T F^{-1}\right)^{l_{1}+l_{2}} \int \frac{\mathrm{d} u_{1} \mathrm{~d} u_{2}}{(2 \pi)^{2}} & T^{\gamma_{l_{1}}\left(u_{1}\right)+\gamma_{l_{2}}\left(u_{2}\right)} S^{\mathrm{i} p_{l_{1}}\left(u_{1}\right)+\mathrm{i} p_{l_{2}}\left(u_{2}\right)} \\
& \times \frac{\mu_{l_{1}}\left(u_{1}\right) \mu_{l_{2}}\left(u_{2}\right) h_{-l_{1}}\left(u_{1}\right) h_{-l_{2}}\left(u_{2}\right)}{P_{l_{1} \mid l_{2}}\left(u_{1} \mid u_{2}\right) P_{l_{2} \mid l_{1}}\left(u_{2} \mid u_{1}\right)} .
\end{aligned}
$$

In the above expressions, $p$ and $\gamma$ denote the momentum and quantum energy correction of the gluon excitations, whereas $\mu, P$ and $h$ are further physical quantities describing them which are known as the measure, pentagon transition and NMHV form factor. They have been derived at finite coupling in [13], and to leading quantum order, their perturbative expansions read

$$
\begin{aligned}
p_{l}(u) & =2 u+2 \mathrm{i} g^{2}\left(\psi\left(\mathrm{i} u^{-}\right)-\psi\left(-\mathrm{i} u^{+}\right)\right)+\mathcal{O}\left(g^{4}\right) \\
\gamma_{l}(u) & =2 g^{2}\left(\psi\left(1+\mathrm{i} u^{-}\right)+\psi\left(1-\mathrm{i} u^{+}\right)-2 \psi(1)\right)+\mathcal{O}\left(g^{4}\right) \\
\mu_{l}(u) & =(-1)^{l} g^{2}\left(u^{+} u^{-}\right)^{-2} \times \frac{\Gamma\left(1+\mathrm{i} u^{-}\right) \Gamma\left(1-\mathrm{i} u^{+}\right)}{\Gamma(l)}+\mathcal{O}\left(g^{4}\right) \\
P_{l_{1} \mid l_{2}}\left(u_{1} \mid u_{2}\right) & =\frac{(-1)^{l_{2}}}{g^{2}} u_{1}^{+} u_{1}^{-} u_{2}^{+} u_{2}^{-} \times \frac{\Gamma\left(-\mathrm{i} u_{1}^{+}+\mathrm{i} u_{2}^{-}\right)}{\Gamma\left(1+\mathrm{i} u_{1}^{-}\right) \Gamma\left(1-\mathrm{i} u_{2}^{+}\right)} \times \frac{\Gamma\left(\mathrm{i} u_{1}^{-}-\mathrm{i} u_{2}^{-}\right)}{\Gamma\left(1-\mathrm{i} u_{1}^{+}+\mathrm{i} u_{2}^{+}\right)}+\mathcal{O}\left(g^{4}\right), \\
h_{l}(u) & :=\left(\frac{1}{g^{2}} x\left(u^{+}\right) x\left(u^{-}\right)\right)^{\operatorname{sign}(l)}
\end{aligned}
$$

expressed in terms of the shifted rapidities

$$
u_{k}^{ \pm}:=u_{k} \pm \frac{\mathrm{i} l_{k}}{2} .
$$

and Zhukowski variables

$$
x(u):=\frac{1}{2}\left(u+\sqrt{u^{2}-4 g^{2}}\right)=u+\mathcal{O}\left(g^{2}\right) .
$$

As a consequence, the $L$-loop contribution to the OPE integrals defined in eqs. (4.12) and (4.13) has the general structure

$$
W_{1_{-}}^{(L)}=g^{2 L} \sum_{l_{1} \geq 1}\left(-\frac{T}{S F}\right)^{l_{1}} \int \frac{\mathrm{d} u_{1}}{2 \pi} \frac{S^{2 \mathrm{i} u_{1}^{-}} \mathcal{P}_{1}^{(L)}}{\left(u_{1}^{+} u_{1}^{-}\right)^{2 L-1}} \times \frac{\Gamma\left(1-\mathrm{i} u_{1}^{+}\right) \Gamma\left(1+\mathrm{i} u_{1}^{-}\right)}{\Gamma\left(l_{1}\right)}
$$

for one gluon bound state, and

$$
\begin{gathered}
W_{2_{-}}^{(L)}=g^{2 L} \sum_{l_{1} \geq l_{2} \geq 1} \frac{1}{1+\delta_{l_{1}, l_{2}}}\left(-\frac{T}{S F}\right)^{l_{1}+l_{2}} \int \frac{\mathrm{d} u_{1} \mathrm{~d} u_{2}}{(2 \pi)^{2}} \frac{S^{2 \mathrm{i}\left(u_{1}^{-}+u_{2}^{-}\right) \mathcal{P}_{2}^{(L)}}}{\left(u_{1}^{+} u_{1}^{-} u_{2}^{+} u_{2}^{-}\right)^{2 L-7}} \times \\
\times \frac{\Gamma\left(1-\mathrm{i} u_{1}^{+}\right)^{2} \Gamma\left(1+\mathrm{i} u_{1}^{-}\right)^{2} \Gamma\left(1-\mathrm{i} u_{2}^{+}\right)^{2} \Gamma\left(1+\mathrm{i} u_{2}^{-}\right)^{2}}{\Gamma\left(l_{1}\right) \Gamma\left(l_{2}\right) \Gamma\left(-\mathrm{i} u_{1}^{+}+\mathrm{i} u_{2}^{-}\right) \Gamma\left(\mathrm{i} u_{1}^{-}-\mathrm{i} u_{2}^{+}\right)} \cdot \frac{\Gamma\left(1+\mathrm{i} u_{1}^{+}-\mathrm{i} u_{2}^{+}\right) \Gamma\left(1-\mathrm{i} u_{1}^{+}+\mathrm{i} u_{2}^{+}\right)}{\Gamma\left(\mathrm{i} u_{1}^{-}-\mathrm{i} u_{2}^{-}\right) \Gamma\left(-\mathrm{i} u_{1}^{-}+\mathrm{i} u_{2}^{-}\right)}
\end{gathered}
$$


for two excitations, which becomes non-zero starting from the $L=6$ loop order. Here the $\mathcal{P}_{k}^{(L)}$ symbols collect factors with polynomial dependence on the shifted rapidities $u_{k}^{ \pm}$, the logarithms of kinematical variables $\{\log (T), \log (S)\}$, and on polygamma functions $\left\{\psi^{\bullet}\left(\mp u_{k}^{ \pm}\right), \psi^{\bullet}\left(1 \mp u_{k}^{ \pm}\right)\right\}$, which appear at higher order in the perturbative expansions (4.14)(4.18). For example, the first two non-zero values for the $\mathcal{P}_{1}^{(L)}$ read:

$$
\begin{aligned}
& \mathcal{P}_{1}^{(2)}=1, \\
& \begin{aligned}
\mathcal{P}_{1}^{(3)}= & \frac{7}{2}\left(u_{1}^{-}\right)^{2}+u_{1}^{-} u_{1}^{+}+\frac{7}{2}\left(u_{1}^{+}\right)^{2}-\left(u_{1}^{-} u_{1}^{+}\right)^{2}\left(-\frac{\pi^{2}}{3}+\left(\psi^{(1)}\left(1+\mathrm{i} u_{1}^{-}\right)+\psi^{(1)}\left(1-\mathrm{i} u_{1}^{+}\right)\right)\right. \\
& +\frac{1}{2}\left(\psi\left(1+\mathrm{i} u_{1}^{-}\right)+\psi\left(1-\mathrm{i} u_{1}^{+}\right)\right)^{2}+\frac{1}{2}\left(\psi\left(\mathrm{i} u_{1}^{-}\right)-\psi\left(-\mathrm{i} u_{1}^{+}\right)\right)^{2} \\
& \left.+2\left(\psi\left(1+\mathrm{i} u_{1}^{-}\right)+\psi\left(1-\mathrm{i} u_{1}^{+}\right)\right) \log (T)+2\left(\psi\left(\mathrm{i} u_{1}^{-}\right)-\psi\left(-\mathrm{i} u_{1}^{+}\right)\right) \log (S)\right) \\
& +\gamma_{\mathrm{E}}(\ldots),
\end{aligned}
\end{aligned}
$$

and similarly for $\mathcal{P}_{2}^{(L)}$ :

$$
\begin{aligned}
\mathcal{P}_{2}^{(6)}= & 1, \\
\mathcal{P}_{2}^{(7)}= & \frac{11}{2}\left[\left(u_{1}^{-} u_{1}^{+} u_{2}^{-}\right)^{2}+\left(u_{1}^{-} u_{1}^{+} u_{2}^{+}\right)^{2}+(1 \leftrightarrow 2)\right] \\
+ & u_{1}^{-} u_{1}^{+} u_{2}^{-} u_{2}^{+}\left(u_{1}^{-} u_{1}^{+}+u_{1}^{-} u_{2}^{-}+u_{1}^{-} u_{2}^{+}+u_{1}^{+} u_{2}^{-}+u_{1}^{+} u_{2}^{+}+u_{2}^{-} u_{2}^{+}\right) \\
- & \left(u_{1}^{-} u_{1}^{+} u_{2}^{-} u_{2}^{+}\right)^{2}\left(-\frac{4 \pi^{2}}{3}+\left[\frac{1}{2}\left(\psi\left(1+\mathrm{i} u_{1}^{-}\right)+\psi\left(1-\mathrm{i} u_{1}^{+}\right)\right)^{2}+\frac{1}{2}\left(\psi\left(\mathrm{i} u_{1}^{-}\right)-\psi\left(-\mathrm{i} u_{1}^{+}\right)\right)^{2}\right.\right. \\
& +2\left(\psi^{(1)}\left(1+\mathrm{i} u_{1}^{-}\right)+\psi^{(1)}\left(1-\mathrm{i} u_{1}^{+}\right)\right)-2 \psi\left(\mathrm{i} u_{1}^{-}\right) \psi\left(-\mathrm{i} u_{1}^{+}\right) \\
& \left.+2\left(\psi\left(1+\mathrm{i} u_{1}^{-}\right)+\psi\left(1-\mathrm{i} u_{1}^{+}\right)\right) \log (T)+2\left(\psi\left(\mathrm{i} u_{1}^{-}\right)-\psi\left(-\mathrm{i} u_{1}^{+}\right)\right) \log (S)+(1 \leftrightarrow 2)\right] \\
& -\left(\psi\left(1+\mathrm{i} u_{1}^{-}\right)+\psi\left(1-\mathrm{i} u_{1}^{+}\right)\right)\left(\psi\left(1+\mathrm{i} u_{2}^{-}\right)+\psi\left(1-\mathrm{i} u_{2}^{+}\right)\right) \\
& \left.-\left(\psi\left(\mathrm{i} u_{1}^{-}\right)-\psi\left(-\mathrm{i} u_{1}^{+}\right)\right)\left(\psi\left(\mathrm{i} u_{2}^{-}\right)+\psi\left(-\mathrm{i} u_{2}^{+}\right)\right)\right) \\
+ & \gamma_{\mathrm{E}}(\ldots) .
\end{aligned}
$$

Here the ellipsis (...) hide terms proportional to the Euler-Mascheroni constant $\gamma_{\mathrm{E}}$, which always drop out from the final answer. Note that the last fraction in eq. (4.22) can actually be simplified to just a polynomial [27]:

$$
\frac{\Gamma\left(1+\mathrm{i} u_{1}^{+}-\mathrm{i} u_{2}^{+}\right) \Gamma\left(1-\mathrm{i} u_{1}^{+}+\mathrm{i} u_{2}^{+}\right)}{\Gamma\left(\mathrm{i} u_{1}^{-}-\mathrm{i} u_{2}^{-}\right) \Gamma\left(-\mathrm{i} u_{1}^{-}+\mathrm{i} u_{2}^{-}\right)}=(-1)^{l_{1}-l_{2}+1}\left(\mathrm{i} u_{1}^{+}-\mathrm{i} u_{2}^{+}\right)\left(\mathrm{i} u_{1}^{-}-\mathrm{i} u_{2}^{-}\right),
$$

which means that it does not contribute any additional poles to the integrand.

After the perturbative expansion of eqs. (4.21) and (4.22) is achieved to the desired loop order $L$, our next step is application of the Cauchy's residue theorem: for $S>1$ the contour of $u_{k}$-integration is closed in the upper half-plane and the residues at poles 


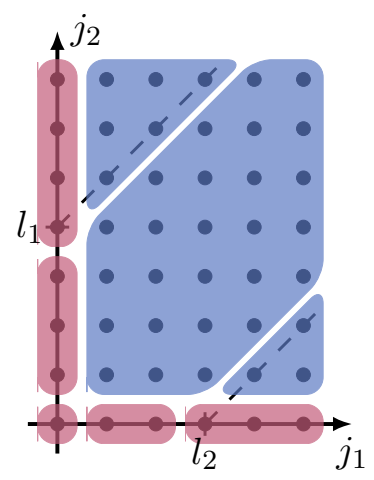

Figure 3. A graphical depiction of the eight regions of the $W_{2_{-}}$summation variables presented on the $\left\{j_{1}, j_{2}\right\}$-plane, parametrized by the helicities $\left\{l_{1}, l_{2}\right\}$ and listed in table 7 .

$u_{k}=\mathrm{i}\left(j_{k}+\frac{l_{k}}{2}\right)$ are collected. Following the ideas of [27, 32], we first change the variables in the integrands according to:

$$
u_{k}=\varepsilon_{k}+\mathrm{i}\left(j_{k}+\frac{l_{k}}{2}\right), \quad u_{k}^{ \pm}=\varepsilon_{k}+\mathrm{i} j_{k}+\left\{\begin{array}{ll}
\mathrm{i} l_{k} & \text { for }+ \\
0 & \text { for }-
\end{array} \text { for } \quad j_{k} \in \mathbb{Z}_{\geq 0} .\right.
$$

Next we expand the integrands in the $\varepsilon_{k} \rightarrow 0$ limit and pick the coefficient of the $\varepsilon_{k}^{-1}$ term, which gives an analytic expression for the residues as functions on an integer lattice of the summation variables $\left\{j_{1}, l_{1}\right\}$ in the one excitation case, and $\left\{j_{1}, j_{2}, l_{1}, l_{2}\right\}$ in the case of two excitations with $j_{k} \geq 0$ and $l_{1} \geq l_{2} \geq 1$.

Special care is needed for the $j_{k}=0$ boundary, where the corresponding shifted rapidity $u_{k}^{-}$in the denominators of the integrands of eqs. (4.21) and (4.22) develop additional $\varepsilon_{k}$ singularities. The lattice of summation variables $\left\{j_{1}, l_{1}\right\}$ gets split into 2 regions with respect to the $j_{1}>0$ condition: one region is $\left\{j_{1}=0\right\}$ and the other is $\left\{j_{1}>0\right\}$. The first two $u$-dependent $\Gamma$-functions in the denominator of eq. (4.22) also require separate treatment, as they reduce the overall $\varepsilon_{k^{-}}$divergence when $-\mathrm{i} u_{1}^{+}+\mathrm{i} u_{2}^{-} \in \mathbb{Z}_{\geq 0}$ or $\mathrm{i} u_{1}^{-}-\mathrm{i} u_{2}^{+} \in \mathbb{Z}_{\geq 0}$. When combined, these conditions split the lattice of summation variables $\left\{j_{1}, j_{2}, l_{1}, l_{2}\right\}$ into 8 regions $\{\mathrm{I}, \ldots, \mathrm{VIII}\}$, as illustrated in figure 3 , which we distinguish by the values of the following 4-tuple of predicates:

$$
\left\{j_{1}>0, j_{2}>0, j_{2}<j_{1}+l_{1}, j_{2}>j_{1}-l_{2}\right\} .
$$

In table 7 we list the eight possible combinations of values of these predicates as well as the total number of the lattice points that satisfy them truncated at $j_{1}+j_{2}+l_{1}+l_{2} \leq N_{\text {opt }}$, where we took the optimal expansion order $N_{\text {opt }}=25$ from table 6 .

Finally we arrive at the sum over residues for the one gluon OPE integral shown in eq. (4.21) containing the following two terms:

$$
W_{1_{-}}^{(L)}=\sum_{j_{1}, l_{1} \geq 1} x^{j_{1}+l_{1}}(1-y)^{j_{1}} \times \frac{\mathcal{Q}_{1}^{(L)}}{\left(j_{1}\left(j_{1}+l_{1}\right)\right)^{2 L-1}} \cdot \frac{\Gamma\left(j_{1}+l_{1}\right)}{\Gamma\left(l_{1}\right) \Gamma\left(1+j_{1}\right)}+\sum_{l_{1} \geq 1} x^{l_{1}} \times \frac{\mathcal{Q}_{2}^{(L)}}{l_{1}^{2 L+2}}
$$




\begin{tabular}{|c|c|c|c|c|c|}
\hline region & $j_{1}>0$ & $j_{2}>0$ & $j_{2}<j_{1}+l_{1}$ & $j_{2}>j_{1}-l_{2}$ & number of points \\
\hline I & $\checkmark$ & $\checkmark$ & $\checkmark$ & $\checkmark$ & 3360 \\
\hline II & $\checkmark$ & $\checkmark$ & $\checkmark$ & $x$ & 2272 \\
\hline III & $\checkmark$ & $\checkmark$ & $x$ & $\checkmark$ & 1232 \\
\hline IV & $\checkmark$ & $x$ & $\checkmark$ & $\checkmark$ & 358 \\
\hline $\mathrm{V}$ & $\checkmark$ & $x$ & $\checkmark$ & $x$ & 864 \\
\hline VI & $x$ & $\checkmark$ & $\checkmark$ & $\checkmark$ & 764 \\
\hline VII & $x$ & $\checkmark$ & $x$ & $\checkmark$ & 458 \\
\hline VIII & $x$ & $x$ & $\checkmark$ & $\checkmark$ & 156 \\
\hline
\end{tabular}

Table 7. The eight regions of the summation variables $\left\{j_{1}, j_{2}, l_{1}, l_{2}\right\}$ and the values of the predicates introduced in eq. (4.29) that separate them. Here we denote $\{\boldsymbol{V}, \boldsymbol{x}\}=\{$ true, false $\}$. The total number of points (or terms in the sums) in each region is evaluated for the expansion order $x^{25}$, which corresponds to $j_{1}+j_{2}+l_{1}+l_{2} \leq N_{\text {opt }}(12)=25$. In figure 3 we also illustrate these regions on the $\left\{j_{1}, j_{2}\right\}$-plane.

where we collected all the dependence on $\left\{j_{1}, l_{1}\right\}$ parameters, as well as $\left\{\psi^{\bullet}\left(j_{1}\right), \psi^{\bullet}\left(j_{1}+l_{1}\right)\right\}$ and the $\{\log (T), \log (S)\}$ logarithms in two factors $\mathcal{Q}_{1}^{(L)}$ for the $j_{1}>0$ region and $\mathcal{Q}_{2}^{(L)}$ for the $j_{1}=0$ region. Similarly, the residue sum for the two gluon OPE integral of eq. (4.22) involves eight terms, one for each region shown in table 7:

$$
\begin{gathered}
W_{2_{-}}^{(L)}=\sum_{\left\{j_{1}, j_{2}, l_{1}, l_{2}\right\} \in \mathrm{I}} x^{j_{1}+j_{2}+l_{1}+l_{2}}(1-y)^{j_{1}+j_{2}} \times \frac{\mathcal{R}_{\mathrm{I}}^{(L)}}{\left(j_{1} j_{2}\left(j_{1}+l_{1}\right)\left(j_{2}+l_{2}\right)\right)^{2 L-6}} \cdot \\
\frac{\Gamma\left(j_{1}+l_{1}\right)^{2} \Gamma\left(j_{2}+l_{2}\right)^{2}}{\Gamma\left(l_{1}\right) \Gamma\left(l_{2}\right) \Gamma\left(1+j_{1}\right)^{2} \Gamma\left(1+j_{2}\right)^{2} \Gamma\left(j_{1}-j_{2}+l_{1}\right) \Gamma\left(-j_{1}+j_{2}+l_{2}\right)}+\text { other regions }\{\mathrm{II}, \ldots, \mathrm{VIII}\}
\end{gathered}
$$

where we hide all the complicated dependence on the summation variables $\left\{j_{1}, j_{2}, l_{1}, l_{2}\right\}$ and the polygamma functions $\left\{\psi^{\bullet}\left(j_{k}\right), \psi^{\bullet}\left(j_{k}+l_{k}\right), \psi^{\bullet}\left(j_{1}-j_{2}+l_{1}\right), \psi^{\bullet}\left(-j_{1}+j_{2}+l_{2}\right)\right\}$ in $\left\{\mathcal{R}_{\mathrm{I}}^{(L)}, \ldots, \mathcal{R}_{\mathrm{VIII}}^{(L)}\right\}$ factors.

While in $[27,32]$ it was shown that the sum representation of the one-gluon contribution (4.30) can be evaluated in terms of MPLs with the help of the algorithms of ref. [39], to our knowledge there exists no direct method for similarly evaluating the two-gluon contribution, eq. (4.13). It is for this reason that we will instead choose to resort to the bootstrap method.

In summary, by virtue of eq. (4.11) the above residue sum representations of the OPE gluonic contributions $W_{1}^{(L)}$ and $W_{2}^{(L)}$ yield the (1111) component of the NMHV Wilson loop $W$ in the DS2 limit as a series expansion around the collinear limit. Converting the latter to the BDS-like normalization according to eq. (4.1), and recalling that it is a pure function as a consequence of eq. (4.10), we then equate it to an ansatz built out of the DS functions we constructed in section 3 , in their series expansion representation (3.18). This fixes all coefficients of our ansatz, and using the MPL representation of our DS functions, we thus determine the (1111) component of $\mathrm{E}^{(L)}$ in this form. 
Before presenting our results in the next section, let us also make some further comments on our computational setup. The form of the sums of eqs. (4.30) and (4.31) indicates that in every term the power of the $x$-variable is bigger than the power of the $y$-variable. In our ansatz, they will thus correspond to the $\mathcal{N}$ "non-diagonal" part of the expansions of eq. (3.18), implying that its "diagonal" part is zero. This makes our ansatz expansion of the form (3.18) well-suited for matching onto eqs. (4.30) and (4.31), and further implies that we may bound the summation ranges of the latter according to $j_{1}+l_{1} \leq N_{\text {opt }}$ and $j_{1}+j_{2}+l_{1}+l_{2} \leq N_{\text {opt }}$, where $N_{\text {opt }}$ is the optimal expansion order discussed in subsection 3.3 and shown in table 6 .

\section{$5 \quad$ Results}

In this work we constructed a space of functions $\mathcal{H}^{\mathrm{DS}}$ relevant for the DS limit of the six gluon scattering amplitude in $\mathcal{N}=4$ super-Yang-Mills theory through transcendental weight $p=12$. We applied the $\mathcal{H}^{\mathrm{DS}}$ space to (partial) calculation of the NMHV component of the six gluon amplitude up to $L \leq 8$ loops.

In section 3.1 we employed extended Steinmann and analyticity conditions in order to construct the coproduct representation of the $\mathcal{H}^{\mathrm{DS}}$ space. In section 3.2 and section 3.3 we present two closely related realizations of the $\mathcal{H}^{\mathrm{DS}}$ : one is in terms of multiple polylogarithms, and one in terms of power-and-log expansions around the $\{u, v, w\} \rightarrow\{0,1,0\}$ kinematical point. The latter is then combined with the residue sum representation of the one and two gluon contributions to the Wilson Loop Operator Product Expansion, which is explained in some detail in section 4. Combination of these two techniques allows us to determine the six gluon NMHV amplitude in the DS limit to high loop order, find new (potentially all-loop) patterns in the analytical form of the amplitude in the $\{u, v, w\} \rightarrow 0$ origin limit, and pinpoint the excessive elements of the $\mathcal{H}^{\mathrm{DS}}$ that should be irrelevant for amplitude calculations as will be explained next.

\subsection{The NMHV amplitude up to 8 loops and weight 12}

As the main application of our DS space of functions $\mathcal{H}^{\mathrm{DS}}$, we have computed the (1111) component of the NMHV superamplitude $\mathrm{E}^{(L)}$ in the double scaling limit $w \rightarrow 0$, more precisely one of its two parity images defined in eq. (4.7). This quantity has the following natural decomposition,

$$
\mathrm{E}^{(L)}(u, v, w) \stackrel{(1111)}{\longrightarrow} \sum_{p=0}^{2 L} \mathrm{E}_{p}^{(L)}(u, v) \cdot(\log (w))^{2 L-p},
$$

where each coefficient $\mathrm{E}_{p}^{(L)}(u, v)$ of a large logarithm $\log (w)^{2 L-p}$ is a pure transcendental function of weight $p$. In particular, we have determined $\mathrm{E}_{p}^{(L)}$ for any $L \leq 8$ and $p \leq 12$, namely the full component for $L \leq 6$, as well as all terms with two (four) or more powers of $\log (w)$ at $L=7(L=8)$. The $p \leq 12$ restriction is due to the maximal weight we have explicit MPL representations for $\mathcal{H}^{\mathrm{DS}}$ so far, whereas the $L \leq 8$ restriction is due to the size of the expressions predicted by the Wilson loop OPE. Nevertheless, we are hopeful 
that the new results we have obtained will offer valuable boundary data and consistency checks for NMHV hexagon in general kinematics at seven [88] and eight loops. We provide expressions $\sum_{L=0}^{6} \sum_{p=0}^{2 L} g^{2 L} \mathrm{E}_{p}^{(L)} \cdot(\log (w))^{2 L-p}$ and $\sum_{L=7}^{8} \sum_{p=0}^{12} g^{2 L} \mathrm{E}_{p}^{(L)} \cdot(\log (w))^{2 L-p}$ for the full (1111) component of the NMHV amplitude in the DS limit up to 6 loops and partial results at 7 and 8 loops in the files EDS-L1-8-w0-12.m.

Let us also describe the checks we have performed on our answers. First off, we confirm BDS-normalized component vanishes in the soft limit boundary $x \rightarrow 0$ limit or, equivalently,

$$
\mathrm{E}^{(L)} \underset{\text { soft \& DS2 }}{\longrightarrow}\left(e^{\frac{1}{4} \Gamma_{\text {cusp }} \mathcal{E}^{(1)}}\right)^{(L)}
$$

as expected. Furthermore, we find perfect agreement with existing results on the collinear limit expansion of the NMHV amplitude, namely the $\left\{T, T^{2}, T^{3}\right\}$ terms for $L \leq 4$ from eEEtT3.m of [50], as well as the first two $\left\{T, T^{2}\right\}$ terms for $L \leq 6$ from W1111L0-6.m of [53] (this time for the individual OPE contributions $W_{1_{-}}^{(L)}$ and $W_{2-}^{(L)}$ ). Additional checks were also performed in an interesting boundary point of the DS limit, that we discuss next.

\subsection{The origin limit}

The origin limit $\{u, v, w\} \rightarrow 0$ was first analyzed in [53], where it was observed that at weak coupling the MHV six-particle amplitude takes a very simple (Sudakov-like) form of exponentiated double logarithms,

$$
\log (\mathcal{E})=-\frac{\Gamma_{\text {oct }}}{24} l_{u v w}^{2}-\frac{\Gamma_{\mathrm{hex}}}{24}\left(l_{u / v}^{2}+l_{v / w}^{2}+l_{w / u}^{2}\right)+C_{0}+\mathcal{O}(u)
$$

in terms of certain quantities $\left\{\Gamma_{\text {oct }}, \Gamma_{\text {hex }}, C_{0}\right\}$ that only depend on the coupling. More recently these quantities have been conjectured at finite coupling [62], based on a Wilson loop OPE resummation procedure morally similar to the one considered in this paper, and backed by further strong coupling analysis via the gauge/string duality.

Although in the latter paper it was also pointed out that the NMHV amplitude no longer displays this exponentiation of double logarithms, it may still be interesting to look for patterns that may hint to an all-loop description. Clearly, the origin limit is a boundary of the DS limit, corresponding to $y \rightarrow 1^{+}, x \rightarrow-\infty$ in the variables (2.26) underlying the construction of our DS function space. We have indeed taken this limit of the general result 
discussed in the previous section, and through four loops it reads

$$
\begin{aligned}
\mathrm{E} & \frac{(1111)}{\mathrm{DS} 2 \& \text { origin }} 1+g^{2}\left[-2 \zeta_{2}+l_{u} l_{v}-\frac{l_{u}^{2}}{2}-\frac{l_{v}^{2}}{2}-\frac{l_{w}^{2}}{2}\right] \\
+ & g^{4}\left[\frac{l_{w}^{4}}{8}+l_{w}^{2}\left(2 \zeta_{2}-\frac{l_{u} l_{v}}{2}+\frac{l_{u}^{2}}{4}+\frac{l_{v}^{2}}{4}\right)\right. \\
& \left.-\frac{1}{2} l_{u}^{3} l_{v}+\frac{1}{2} l_{u}^{2} l_{v}^{2}-\frac{1}{2} l_{u} l_{v}^{3}+\frac{l_{u}^{4}}{8}+\frac{3}{2} \zeta_{2} l_{v}^{2}+\zeta_{3} l_{v}+\frac{l_{v}^{4}}{8}+\frac{39 \zeta_{2}^{2}}{10}+\frac{3}{2} \zeta_{2} l_{u}^{2}+\zeta_{3} l_{u}-5 \zeta_{2} l_{u} l_{v}\right] \\
+ & g^{6}\left[-\frac{l_{w}^{6}}{48}+l_{w}^{4}\left(-\frac{3 \zeta_{2}}{4}+\frac{l_{u} l_{v}}{8}-\frac{l_{u}^{2}}{16}-\frac{l_{v}^{2}}{16}\right)\right. \\
& +l_{w}^{2}\left(-\frac{167 \zeta_{2}^{2}}{20}-\frac{5}{4} \zeta_{2} l_{u}^{2}-\frac{\zeta_{3} l_{u}}{2}+\frac{7}{2} \zeta_{2} l_{u} l_{v}+\frac{1}{4} l_{u}^{3} l_{v}-\frac{1}{4} l_{u}^{2} l_{v}^{2}+\frac{1}{4} l_{u} l_{v}^{3}-\frac{l_{u}^{4}}{16}-\frac{5}{4} \zeta_{2} l_{v}^{2}-\frac{\zeta_{3} l_{v}}{2}-\frac{l_{v}^{4}}{16}\right) \\
& +l_{w}\left(\frac{1}{2} \zeta_{3} l_{u}^{2}+2 \zeta_{2}^{2} l_{u}+\frac{1}{2} \zeta_{2} l_{u}^{2} l_{v}+\frac{1}{2} \zeta_{2} l_{u} l_{v}^{2}+2 \zeta_{2}^{2} l_{v}+\frac{1}{2} \zeta_{3} l_{v}^{2}\right) \\
& +\frac{1}{8} l_{u}^{5} l_{v}-\frac{3}{16} l_{u}^{4} l_{v}^{2}+\frac{5}{18} l_{u}^{3} l_{v}^{3}-\frac{3}{16} l_{u}^{2} l_{v}^{4}+\frac{1}{8} l_{u} l_{v}^{5}-\frac{l_{u}^{6}}{48}-\frac{69}{20} \zeta_{2}^{2} l_{v}^{2}-\frac{1}{2} \zeta_{2} l_{v}^{4}-\zeta_{3} l_{v}^{3}-6 \zeta_{2} \zeta_{3} l_{v}-8 \zeta_{5} l_{v}-\frac{l_{v}^{6}}{48} \\
& \left.-\frac{527 \zeta_{2}^{3}}{105}-\frac{1}{2} \zeta_{2} l_{u}^{4}-\zeta_{3} l_{u}^{3}-\frac{69}{20} \zeta_{2}^{2} l_{u}^{2}-6 \zeta_{2} \zeta_{3} l_{u}-8 \zeta_{5} l_{u}+\frac{19}{6} \zeta_{2} l_{u}^{3} l_{v}-\frac{5}{2} \zeta_{2} l_{u}^{2} l_{v}^{2}+\frac{271}{10} \zeta_{2}^{2} l_{u} l_{v}+\frac{19}{6} \zeta_{2} l_{u} l_{v}^{3}\right] \\
+ & \mathcal{O}\left(g^{8}\right),
\end{aligned}
$$

where the symbol $l_{x}$ represents a simple logarithm: $l_{x}:=\log (x)$ as defined in eq. (3.14). Higher loop order $L \leq 8$ corrections to eq. (5.4) bounded by transcendental weight $p \leq 12$ can be found in the ancillary file EDS-origin-L1-8-w0-12.m.

In this manner, we confirm that no additional cancellations occur for the (1111) component, that would lead to a Sudakov-like form similar to the MHV amplitude at the origin. We have also further vetted our result by comparing it with the origin limit of the NMHV amplitude in general kinematics through six loops [88, 89], finding perfect agreement.

While it may be worthwhile to also look at other components for additional cancellations, already from the current data we observe an interesting general pattern for the three highest powers of divergent logarithms for the NMHV/MHV ratio function. In particular, up to $L \leq 6$ loops we find ${ }^{8}$

$$
\begin{aligned}
\mathrm{E} / \mathcal{E} & \underset{\mathrm{DS} 2 \& \text { origin }}{\longrightarrow} \sum_{L=0}^{6} g^{2 L}\left[\frac{\left(l_{u} l_{v}\right)^{L}}{(L !)^{2}}\right. \\
& +\zeta_{2}\left(-\frac{\left(l_{u} l_{v}\right)^{L-2}\left(l_{u}+l_{v}\right) l_{w}}{(L-2) !(L-1) !}-\frac{\left(l_{u} l_{v}\right)^{L-2}\left((L-1)^{2} l_{u}^{2}+\left(4(L-1)^{2}+3(L-1)-1\right) l_{u} l_{v}+(L-1)^{2} l_{v}^{2}\right)}{(L-1) ! L !}\right) \\
& \left.+\zeta_{3}\left(\frac{\left(l_{u} l_{v}\right)^{L-3}\left(l_{u}^{2}+l_{v}^{2}\right) l_{w}}{(L-2) ! L !}-\frac{\left(l_{u} l_{v}\right)^{L-3}\left(l_{u}+l_{v}\right)\left((L-2) l_{u}^{2}-(L-1) l_{u} l_{v}+(L-2) l_{v}^{2}\right)}{(L-2) !(L-1) !}\right)+\mathcal{O}\left(\left(l_{u} l_{v}\right)^{2 L-4}\right)\right] \\
& +\mathcal{O}\left(g^{14}\right),
\end{aligned}
$$

It may thus well be that this pattern persists at higher loops, especially given that the two-gluon OPE excitation already contributes at $L=6$ loops in the DS limit.

\footnotetext{
${ }^{8}$ We acknowledge discussions with Lance Dixon, who first noticed the leading $1 /(L !)^{2}$ behavior on the $u=v=w$ line of the NMHV amplitude at the origin, motivating us to carry out the following analysis.
} 


\subsection{Further refinements of the $\mathcal{H}^{\mathrm{DS}}$ space}

In this final subsection, let us also discuss how it may be possible to further reduce the size of the $\mathcal{H}^{\mathrm{DS}}$ functional space, so as to make bootstrapping at higher loops more tractable. Our construction of $\mathcal{H}^{\mathrm{DS}}$ includes the entire basis of independent MZVs from eq. (A.1), while we notice only the ordinary zeta values appear in the NMHV remainder E function. This is an indication that perhaps only the latter are needed as independent constants in our space.

To answer such questions about the potential redundancy of our space, we studied the "nested derivatives" or the $\{n, 1, \ldots, 1\}$ coproduct components of the NMHV amplitude expressed in terms of the elements of the $\mathcal{H}^{\mathrm{DS}}$ space, following the blueprint of [54]. In table 8 we report our findings on the dimensions of the minimal subspaces, needed to match the $\mathrm{E}_{p}^{(6)}$ and its coproducts. Note how these dimensions saturate for increasing weight $p$ and fixed $n^{\text {th }}$ coproduct component for

$$
p=2 n+2
$$

at the latest, and the saturated value is smaller than the dimension of the corresponding DS subspace $\operatorname{dim}_{\mathbb{Q}} \mathcal{H}_{n}^{\mathrm{DS}}$, which implies existence of unnecessary elements in the $\mathcal{H}^{\mathrm{DS}}$ space. We find that the first such "extra" function $f_{1}^{(3) \text {,extra }} \in \mathcal{H}_{3}^{\text {DS }}$ at weight $p=3$ that does not appear in the NMHV amplitude reads:

$$
f_{1}^{(3), \text { extra }}=f_{2}^{(3)} \equiv f_{1}^{(1)} \zeta_{2}=l_{1-x y} \zeta_{2},
$$

where $\check{y}:=\frac{1}{y}, \mathcal{G}_{\vec{X}}:=G(\vec{X} ; x)$, and $l_{x}:=\log (x)$. Similarly, at weight $p=4$ the space of "extra" functions is spanned by the following four elements:

$$
\begin{aligned}
f_{1}^{(4), \text { extra }} & =f_{1}^{(1)} \zeta_{3}=l_{1-x y} \zeta_{3}, \\
f_{2}^{(4), \text { extra }} & =f_{1}^{(2)} \zeta_{2}=\mathcal{G}_{0 \check{y}} \zeta_{2}, \\
f_{3}^{(4), \text { extra }} & =f_{2}^{(2)} \zeta_{2}=\left(\mathcal{G}_{0 \check{y}}-\mathcal{G}_{1 \check{y}}-\mathcal{G}_{01}+l_{1-x} l_{x(1-y)}\right) \zeta_{2}, \\
f_{4}^{(4), \text { extra }} & =f_{4}^{(2)} \zeta_{2}=\left(l_{x(1-y)} l_{1-x y}-l_{1-x y}^{2}\right) \zeta_{2},
\end{aligned}
$$

and at weight $p=5$ we found the following eleven functions:

$$
\begin{array}{r}
\left\{f_{1}^{(5), \text { extra }}, \ldots, f_{11}^{(5), \text { extra }}\right\}=\left\{f_{1}^{(3)} \zeta_{2}, f_{1}^{(2)} \zeta_{3}, f_{4}^{(3)} \zeta_{2}, f_{5}^{(3)} \zeta_{2}, f_{2}^{(2)} \zeta_{3}, f_{9}^{(3)} \zeta_{2},\right. \\
\left.f_{7}^{(3)} \zeta_{2}, f_{8}^{(3)} \zeta_{2}, f_{10}^{(3)} \zeta_{2} f_{4}^{(2)} \zeta_{3}, f_{11}^{(3)} \zeta_{2}\right\}
\end{array}
$$

Interestingly, we see that while $\zeta_{2}$ and $\zeta_{3}$ are needed as independent constants in our space, not all of their products with non-constant DS functions appear at higher weight. From these findings we conclude that our DS functional space $\mathcal{H}^{\mathrm{DS}}$ is indeed overcomplete, at least for the problem of bootstrapping the NMHV remainder function in $\mathcal{N}=4 \mathrm{SYM}$. It would be interesting to perform a more careful study of the intricate interplay between the integrability and extended Steinmann relations of eqs. (2.31) and (2.32), the branch cut conditions of eq. (2.38), and the coaction principle [54] in order to further perfect the DS bootstrap. We hope that it will provide valuable insights useful for the general hexagon bootstrap program as well as for other computational problems in high energy physics. 


\begin{tabular}{|l|ccccccccccc|}
\hline$n^{\text {th }}$ comp. & 1 & 2 & 3 & 4 & 5 & 6 & 7 & 8 & 9 & 10 & 11 \\
\hline$p=2$ & 1 & & & & & & & & & & \\
$p=3$ & 1 & 1 & & & & & & & & & \\
$p=4$ & 2 & 4 & 2 & & & & & & & & \\
$p=5$ & 2 & 4 & 5 & 2 & & & & & & & \\
$p=6$ & 2 & 5 & 9 & 5 & 2 & & & & & & \\
$p=7$ & 2 & 5 & 10 & 11 & 5 & 2 & & & & & \\
$p=8$ & 2 & 5 & 11 & 19 & 12 & 5 & 2 & & & & \\
$p=9$ & 2 & 5 & 11 & 22 & 28 & 12 & 5 & 2 & & & \\
$p=10$ & 2 & 5 & 11 & 22 & 40 & 28 & 12 & 5 & 2 & & \\
$p=11$ & 2 & 5 & 11 & 22 & 44 & 60 & 28 & 12 & 5 & 2 & \\
$p=12$ & 2 & 5 & 11 & 22 & 45 & 79 & 60 & 28 & 12 & 5 & 2 \\
\hline functions & 2 & 5 & 12 & 26 & 56 & 116 & 236 & 474 & 943 & 1867 & 3686 \\
\hline
\end{tabular}

Table 8. The number of independent $\{n, 1, \ldots, 1\}$ coproduct components of all $\log w$ coefficients $\mathrm{E}_{p}^{(6)}$ of the $L=6$ loop NMHV amplitude from eq. (5.2). For the reader's convenience we copied the total number of DS functions $f^{(p)}$ at each weight $p$ from table 2 .

\section{Acknowledgments}

GP would like to thank Benjamin Basso, Simon Caron-Huot, Lance Dixon, Falko Dulat, Matt von Hippel and Andrew McLeod for collaboration on closely related topics, as well as Lance Dixon and Ömer Gürdoğan for discussions on related $A_{2}$ function counts. VC thanks Sven-Olaf Moch for helpful discussions and encouragement. The authors acknowledge support from the Deutsche Forschungsgemeinschaft (DFG) under Germany's Excellence Strategy EXC 2121 "Quantum Universe" 390833306. The work of VC has also been supported by the DFG under grant number MO 1801/2-1.

\section{A The multiple zeta value basis}

In this work we used the basis and reduction rules for MZVs from the MZV datamine project [86]. The basis reads as follows:

$$
\begin{array}{r}
\left\{\left\{\zeta_{2}\right\},\left\{\zeta_{3}\right\},\left\{\zeta_{2}^{2}\right\},\left\{\zeta_{2} \zeta_{3}, \zeta_{5}\right\},\left\{\zeta_{2}^{3}, \zeta_{3}^{2}\right\},\left\{\zeta_{2}^{2} \zeta_{3}, \zeta_{2} \zeta_{5}, \zeta_{7}\right\},\left\{\zeta_{2}^{4}, \zeta_{2} \zeta_{3}^{2}, \zeta_{3} \zeta_{5}, \zeta_{5,3}\right\}\right. \\
\left\{\zeta_{2}^{3} \zeta_{3}, \zeta_{3}^{3}, \zeta_{2}^{2} \zeta_{5}, \zeta_{2} \zeta_{7}, \zeta_{9}\right\},\left\{\zeta_{2}^{5}, \zeta_{2}^{2} \zeta_{3}^{2}, \zeta_{2} \zeta_{3} \zeta_{5}, \zeta_{5}^{2}, \zeta_{3} \zeta_{7}, \zeta_{2} \zeta_{5,3}, \zeta_{7,3}\right\} \\
\left\{\zeta_{11}, \zeta_{2}^{4} \zeta_{3}, \zeta_{2} \zeta_{3}^{3}, \zeta_{2}^{3} \zeta_{5}, \zeta_{3}^{2} \zeta_{5}, \zeta_{3} \zeta_{5,3}, \zeta_{5,3,3}, \zeta_{2}^{2} \zeta_{7}, \zeta_{2} \zeta_{9}\right\} \\
\left.\left\{\zeta_{2}^{6}, \zeta_{2}^{3} \zeta_{3}^{2}, \zeta_{3}^{4}, \zeta_{2}^{2} \zeta_{3} \zeta_{5}, \zeta_{2} \zeta_{5}^{2}, \zeta_{2}^{2} \zeta_{5,3}, \zeta_{6,4,1,1}, \zeta_{2} \zeta_{3} \zeta_{7}, \zeta_{5} \zeta_{7}, \zeta_{2} \zeta_{7,3}, \zeta_{3} \zeta_{9}, \zeta_{9,3}\right\}\right\}
\end{array}
$$




\section{B Tensor perspective on the coproduct bootstrap}

Here we give another point of view on the integrability and Steinmann constraints discussed in section 3.1 using tensorial notation. To generate a DS function $f^{(p)} \in \mathcal{H}_{p}^{\mathrm{DS}}$ at weight $p$ let us form an ansatz for the $\{p-1,1\}$ coproduct component:

$$
\Delta_{p-1,1}\left(f^{(p)}\right)=\sum_{j, \alpha} c_{j \alpha} f_{j}^{(p-1)} \otimes \log (\alpha)
$$

with unknown rational coefficients $c_{j \alpha} \in \mathbb{Q}^{\left|J_{p-1}\right| \times|\mathcal{A}|}$. Next we apply eq. (3.5) to functions $f_{j}^{(p-1)}$ of the previous weight, and introduce a set of integrability and extended Steinmann conditions $\mathcal{S}$ on the $\{p-2,1,1\}$ component of the produced coproduct shown in eqs. (2.31) and (2.32) (see also the discussion around eq. (3.7)). For practical purposes, we collect these constraints in a single tensor $\mathcal{I}_{\alpha \beta s} \in \mathbb{Q}^{|\mathcal{A}| \times|\mathcal{A}| \times|\mathcal{S}|}$, whose non-zero elements are summarized below:

$$
\begin{aligned}
\mathcal{I}_{1,1,5} & =-\mathcal{I}_{1,2,5}=\frac{1}{2} \mathcal{I}_{1,3,5}=\mathcal{I}_{1,4,5}=\mathcal{I}_{1,5,5}=1, \\
\mathcal{I}_{2,3,4} & =-\mathcal{I}_{2,4,3}=-\mathcal{I}_{2,4,5}=\mathcal{I}_{2,5,4}=1, \\
-\mathcal{I}_{3,3,4} & =-\mathcal{I}_{3,3,5}=\mathcal{I}_{3,4,3}=\mathcal{I}_{3,5,3}=1, \\
\mathcal{I}_{4,1,5} & =-\mathcal{I}_{4,2,5}=-\mathcal{I}_{4,3,4}=\mathcal{I}_{4,4,3}=-\mathcal{I}_{4,5,1}=\mathcal{I}_{4,5,2}=1, \\
-\mathcal{I}_{5,1,4} & =\mathcal{I}_{5,4,1}=\mathcal{I}_{6,3,2}=\mathcal{I}_{7,2,3}=-\mathcal{I}_{8,1,2}=\mathcal{I}_{8,2,1}=1 .
\end{aligned}
$$

The action of these conditions on the $\{p-2,1,1\}$ coproduct component of the unknown function $f^{(p)}$ follow from eq. (B.1) and read:

$$
\sum_{\substack{j, k \\ \alpha, \beta}} c_{j \alpha} c_{j k \beta}^{(p-1)} f_{k}^{(p-2)} \mathcal{I}_{s \alpha \beta}=\sum_{\substack{j, k \\ \alpha}} f_{k}^{(p-2)} M_{k s j \alpha} c_{j \alpha}=0, \quad \text { for each } s \in \mathcal{S},
$$

where $M_{k s j \alpha} \in \mathbb{Q}^{\left|J_{p-2}\right| \times|\mathcal{S}| \times\left|J_{p-1}\right| \times|\mathcal{A}|}$ (implying the necessary transposition of tensor indices here) was already defined in eq. (3.8) above. Now note, that the DS functions of lower weight $f_{k}^{(p-2)}$ are linearly independent from one another by construction, which means that eq. (B.3) contains $\left|J_{p-2}\right| \cdot|\mathcal{S}|$ constraints for $\left|J_{p-1}\right| \cdot|\mathcal{A}|$ unknowns. The space of DS functions at weight $p$ lies inside the nullspace of the $M_{(k s)(j \alpha)} \in \mathbb{Q}^{\left(\left|J_{p-2}\right| \cdot|\mathcal{S}|\right) \times\left(\left|J_{p-1}\right| \cdot|\mathcal{A}|\right)}$ matrix: schematically we write $\mathcal{H}_{p}^{\mathrm{DS}} \subset \operatorname{Ker}\left(M_{(k s)(j \alpha)}\right)$, where the brackets $(k s)$ and $(j \alpha)$ denote vectorization of indices. In MATHEMATicA such a vectorization $M_{k s j \alpha} \rightarrow M_{(k s)(j \alpha)}$ of a rank 4 tensor into a rank 2 one is achieved simply via application of Flatten [\#, $\{\{1$, $2\},\{3,4\}\}] \&$. The further processing of this nullspace is described in the main text in section 3.1 .

Open Access. This article is distributed under the terms of the Creative Commons Attribution License (CC-BY 4.0), which permits any use, distribution and reproduction in any medium, provided the original author(s) and source are credited. 


\section{References}

[1] L. Brink, J.H. Schwarz and J. Scherk, Supersymmetric Yang-Mills Theories, Nucl. Phys. B 121 (1977) 77 [INSPIRE].

[2] F. Gliozzi, J. Scherk and D.I. Olive, Supersymmetry, Supergravity Theories and the Dual Spinor Model, Nucl. Phys. B 122 (1977) 253 [InSPIRE].

[3] G. 't Hooft, A Planar Diagram Theory for Strong Interactions, Nucl. Phys. B 72 (1974) 461 [INSPIRE].

[4] N. Beisert et al., Review of AdS/CFT Integrability: An Overview, Lett. Math. Phys. 99 (2012) 3 [arXiv: 1012.3982] [INSPIRE].

[5] F. Levkovich-Maslyuk, A review of the AdS/CFT Quantum Spectral Curve, J. Phys. A 53 (2020) 283004 [arXiv: 1911.13065] [INSPIRE].

[6] L.F. Alday and J.M. Maldacena, Gluon scattering amplitudes at strong coupling, JHEP 06 (2007) 064 [arXiv: 0705.0303] [INSPIRE].

[7] J.M. Drummond, G.P. Korchemsky and E. Sokatchev, Conformal properties of four-gluon planar amplitudes and Wilson loops, Nucl. Phys. B 795 (2008) 385 [arXiv:0707.0243] [INSPIRE].

[8] A. Brandhuber, P. Heslop and G. Travaglini, $M H V$ amplitudes in $N=4$ super Yang-Mills and Wilson loops, Nucl. Phys. B $\mathbf{7 9 4}$ (2008) 231 [arXiv:0707.1153] [InSPIRE].

[9] L.F. Alday, D. Gaiotto, J. Maldacena, A. Sever and P. Vieira, An Operator Product Expansion for Polygonal null Wilson Loops, JHEP 04 (2011) 088 [arXiv: 1006.2788] [INSPIRE].

[10] B. Basso, A. Sever and P. Vieira, Spacetime and Flux Tube S-Matrices at Finite Coupling for $N=4$ Supersymmetric Yang-Mills Theory, Phys. Rev. Lett. 111 (2013) 091602 [arXiv: 1303.1396] [INSPIRE].

[11] B. Basso, A. Sever and P. Vieira, Space-time S-matrix and Flux tube S-matrix II. Extracting and Matching Data, JHEP 01 (2014) 008 [arXiv:1306.2058] [INSPIRE].

[12] B. Basso, A. Sever and P. Vieira, Space-time S-matrix and Flux-tube S-matrix III. The two-particle contributions, JHEP 08 (2014) 085 [arXiv: 1402.3307] [INSPIRE].

[13] B. Basso, A. Sever and P. Vieira, Space-time S-matrix and Flux-tube S-matrix IV. Gluons and Fusion, JHEP 09 (2014) 149 [arXiv:1407.1736] [INSPIRE].

[14] A.V. Belitsky, Nonsinglet pentagons and NMHV amplitudes, Nucl. Phys. B 896 (2015) 493 [arXiv: 1407.2853] [INSPIRE].

[15] A.V. Belitsky, Fermionic pentagons and NMHV hexagon, Nucl. Phys. B 894 (2015) 108 [arXiv: 1410.2534] [INSPIRE].

[16] B. Basso, J. Caetano, L. Cordova, A. Sever and P. Vieira, OPE for all Helicity Amplitudes, JHEP 08 (2015) 018 [arXiv: 1412.1132] [INSPIRE].

[17] B. Basso, J. Caetano, L. Cordova, A. Sever and P. Vieira, OPE for all Helicity Amplitudes II. Form Factors and Data Analysis, JHEP 12 (2015) 088 [arXiv: 1508.02987] [INSPIRE].

[18] B. Basso, A. Sever and P. Vieira, Hexagonal Wilson loops in planar $\mathcal{N}=4 S Y M$ theory at finite coupling, J. Phys. A 49 (2016) 41LT01 [arXiv:1508.03045] [INSPIRE].

[19] A.V. Belitsky, Matrix pentagons, Nucl. Phys. B 923 (2017) 588 [arXiv:1607.06555] [INSPIRE]. 
[20] J.M. Drummond, Review of AdS/CFT Integrability, Chapter V.2: Dual Superconformal Symmetry, Lett. Math. Phys. 99 (2012) 481 [arXiv:1012.4002] [INSPIRE].

[21] D. Fioravanti, S. Piscaglia and M. Rossi, Asymptotic Bethe Ansatz on the GKP vacuum as a defect spin chain: scattering, particles and minimal area Wilson loops, Nucl. Phys. B $\mathbf{8 9 8}$ (2015) 301 [arXiv: 1503.08795] [INSPIRE].

[22] A. Bonini, D. Fioravanti, S. Piscaglia and M. Rossi, Strong Wilson polygons from the lodge of free and bound mesons, JHEP 04 (2016) 029 [arXiv: 1511.05851] [INSPIRE].

[23] A. Bonini, D. Fioravanti, S. Piscaglia and M. Rossi, The contribution of scalars to $\mathcal{N}=4$ SYM amplitudes, Phys. Rev. D 95 (2017) 041902 [arXiv: 1607.02084] [InSPIRE].

[24] A. Bonini, D. Fioravanti, S. Piscaglia and M. Rossi, The contribution of scalars to $\mathcal{N}=4$ SYM amplitudes II: Young tableaux, asymptotic factorisation and strong coupling, Nucl. Phys. B 931 (2018) 19 [arXiv:1707.05767] [INSPIRE].

[25] A. Bonini, D. Fioravanti, S. Piscaglia and M. Rossi, Fermions and scalars in $\mathcal{N}=4$ Wilson loops at strong coupling and beyond, Nucl. Phys. B 944 (2019) 114644 [arXiv:1807.09743] [INSPIRE].

[26] S. Caron-Huot, L.J. Dixon, M. von Hippel, A.J. McLeod and G. Papathanasiou, The Double Pentaladder Integral to All Orders, JHEP 07 (2018) 170 [arXiv: 1806.01361] [inSPIRE].

[27] J.M. Drummond and G. Papathanasiou, Hexagon OPE Resummation and Multi-Regge Kinematics, JHEP 02 (2016) 185 [arXiv:1507.08982] [INSPIRE].

[28] L. Córdova, Hexagon POPE: effective particles and tree level resummation, JHEP 01 (2017) 051 [arXiv: 1606.00423] [INSPIRE].

[29] H.T. Lam and M. von Hippel, Resumming the POPE at One Loop, JHEP 12 (2016) 011 [arXiv: 1608.08116] [INSPIRE].

[30] L.V. Bork and A.I. Onishchenko, Pentagon OPE resummation in $N=4$ SYM: hexagons with one effective particle contribution, Phys. Rev. D 102 (2020) 026002 [arXiv:1909.13675] [INSPIRE].

[31] L.V. Bork and A.I. Onishchenko, Pentagon OPE Resummation in $N=4$ SYM: One Effective Particle and MHV Amplitude, Phys. Part. Nucl. 51 (2020) 531 [INSPIRE].

[32] G. Papathanasiou, Hexagon Wilson Loop OPE and Harmonic Polylogarithms, JHEP 11 (2013) 150 [arXiv:1310.5735] [INSPIRE].

[33] G. Papathanasiou, Evaluating the six-point remainder function near the collinear limit, Int. J. Mod. Phys. A 29 (2014) 1450154 [arXiv:1406.1123] [InSPIRE].

[34] D. Gaiotto, J. Maldacena, A. Sever and P. Vieira, Pulling the straps of polygons, JHEP 12 (2011) 011 [arXiv:1102.0062] [INSPIRE].

[35] Z. Bern, L.J. Dixon and V.A. Smirnov, Iteration of planar amplitudes in maximally supersymmetric Yang-Mills theory at three loops and beyond, Phys. Rev. D 72 (2005) 085001 [hep-th/0505205] [INSPIRE].

[36] N. Arkani-Hamed, J.L. Bourjaily, F. Cachazo, A.B. Goncharov and A. Postnikov and J. Trnka, Grassmannian Geometry of Scattering Amplitudes, Cambridge University Press (2016) [DOI].

[37] J. Golden, A.B. Goncharov, M. Spradlin, C. Vergu and A. Volovich, Motivic Amplitudes and Cluster Coordinates, JHEP 01 (2014) 091 [arXiv: 1305.1617] [INSPIRE]. 
[38] J. Drummond, J. Foster and O. Gürdoğan, Cluster adjacency beyond MHV, JHEP 03 (2019) 086 [arXiv: 1810.08149 ] [INSPIRE].

[39] S. Moch, P. Uwer and S. Weinzierl, Nested sums, expansion of transcendental functions and multiscale multiloop integrals, J. Math. Phys. 43 (2002) 3363 [hep-ph/0110083] [INSPIRE].

[40] T. Gehrmann and E. Remiddi, Two loop master integrals for $\gamma * \rightarrow 3$ jets: The Planar topologies, Nucl. Phys. B 601 (2001) 248 [hep-ph/0008287] [INSPIRE].

[41] C. Schneider, Symbolic summation assists combinatorics, Sem. Lothar. Combin. 56 (2007) 1.

[42] L.J. Dixon, C. Duhr and J. Pennington, Single-valued harmonic polylogarithms and the multi-Regge limit, JHEP 10 (2012) 074 [arXiv:1207.0186] [INSPIRE].

[43] L.J. Dixon, J.M. Drummond and J.M. Henn, Bootstrapping the three-loop hexagon, JHEP 11 (2011) 023 [arXiv: 1108.4461] [INSPIRE].

[44] L.J. Dixon, J.M. Drummond and J.M. Henn, Analytic result for the two-loop six-point NMHV amplitude in $N=4$ super Yang-Mills theory, JHEP 01 (2012) 024 [arXiv:1111.1704] [INSPIRE].

[45] L.J. Dixon, J.M. Drummond, M. von Hippel and J. Pennington, Hexagon functions and the three-loop remainder function, JHEP 12 (2013) 049 [arXiv:1308.2276] [INSPIRE].

[46] L.J. Dixon, J.M. Drummond, C. Duhr and J. Pennington, The four-loop remainder function and multi-Regge behavior at NNLLA in planar $N=4$ super-Yang-Mills theory, JHEP 06 (2014) 116 [arXiv: 1402.3300] [INSPIRE].

[47] L.J. Dixon and M. von Hippel, Bootstrapping an NMHV amplitude through three loops, JHEP 10 (2014) 065 [arXiv: 1408.1505] [INSPIRE].

[48] J.M. Drummond, G. Papathanasiou and M. Spradlin, A Symbol of Uniqueness: The Cluster Bootstrap for the 3-Loop MHV Heptagon, JHEP 03 (2015) 072 [arXiv:1412.3763] [INSPIRE].

[49] L.J. Dixon, M. von Hippel and A.J. McLeod, The four-loop six-gluon NMHV ratio function, JHEP 01 (2016) 053 [arXiv: 1509.08127] [INSPIRE].

[50] S. Caron-Huot, L.J. Dixon, A. McLeod and M. von Hippel, Bootstrapping a Five-Loop Amplitude Using Steinmann Relations, Phys. Rev. Lett. 117 (2016) 241601 [arXiv: 1609.00669] [INSPIRE].

[51] L.J. Dixon, J. Drummond, T. Harrington, A.J. McLeod, G. Papathanasiou and M. Spradlin, Heptagons from the Steinmann Cluster Bootstrap, JHEP 02 (2017) 137 [arXiv:1612.08976] [INSPIRE].

[52] J. Drummond, J. Foster, O. Gürdoğan and G. Papathanasiou, Cluster adjacency and the four-loop NMHV heptagon, JHEP 03 (2019) 087 [arXiv:1812.04640] [INSPIRE].

[53] S. Caron-Huot, L.J. Dixon, F. Dulat, M. von Hippel, A.J. McLeod and G. Papathanasiou, Six-Gluon amplitudes in planar $\mathcal{N}=4$ super-Yang-Mills theory at six and seven loops, JHEP 08 (2019) 016 [arXiv: 1903.10890] [inSPIRE].

[54] S. Caron-Huot, L.J. Dixon, F. Dulat, M. Von Hippel, A.J. McLeod and G. Papathanasiou, The Cosmic Galois Group and Extended Steinmann Relations for Planar $\mathcal{N}=4$ SYM Amplitudes, JHEP 09 (2019) 061 [arXiv: 1906.07116] [INSPIRE].

[55] L.J. Dixon and Y.-T. Liu, Lifting Heptagon Symbols to Functions, JHEP 10 (2020) 031 [arXiv:2007.12966] [INSPIRE]. 
[56] S. Caron-Huot et al., The Steinmann Cluster Bootstrap for $N=4$ Super Yang-Mills Amplitudes, PoS CORFU2019 (2020) 003 [arXiv: 2005.06735] [InSPIRE].

[57] O. Steinmann, Über den Zusammenhang zwischen den Wightmanfunktionen und der retardierten Kommutatoren, Helv. Phys. Acta 33 (1960) 257.

[58] O. Steinmann, Wightman-Funktionen und retardierten Kommutatoren. II, Helv. Phys. Acta 33 (1960) 347.

[59] K.E. Cahill and H.P. Stapp, Optical theorems and steinmann relations, Annals Phys. 90 (1975) 438 [INSPIRE].

[60] J. Drummond, J. Foster and O. Gürdoğan, Cluster Adjacency Properties of Scattering Amplitudes in $N=4$ Supersymmetric Yang-Mills Theory, Phys. Rev. Lett. 120 (2018) 161601 [arXiv: 1710.10953] [INSPIRE].

[61] C. Duhr, Mathematical aspects of scattering amplitudes, in Theoretical Advanced Study Institute in Elementary Particle Physics: Journeys Through the Precision Frontier: Amplitudes for Colliders, (2014), DOI [arXiv:1411.7538] [INSPIRE].

[62] B. Basso, L.J. Dixon and G. Papathanasiou, Origin of the Six-Gluon Amplitude in Planar $N=4$ Supersymmetric Yang-Mills Theory, Phys. Rev. Lett. 124 (2020) 161603 [arXiv: 2001.05460] [INSPIRE].

[63] N. Arkani-Hamed, J.L. Bourjaily, F. Cachazo, S. Caron-Huot and J. Trnka, The All-Loop Integrand For Scattering Amplitudes in Planar N =4 SYM, JHEP 01 (2011) 041 [arXiv: 1008.2958] [INSPIRE].

[64] A.J. McLeod, H. Munch, G. Papathanasiou and M. von Hippel, A Novel Algorithm for Nested Summation and Hypergeometric Expansions, JHEP 11 (2020) 122 [arXiv:2005.05612] [INSPIRE].

[65] https://gitlab.com/vchestnov/ds-bootstrap.

[66] L.F. Alday, D. Gaiotto and J. Maldacena, Thermodynamic Bubble Ansatz, JHEP 09 (2011) 032 [arXiv:0911.4708] [INSPIRE].

[67] J.M. Drummond, J. Henn, G.P. Korchemsky and E. Sokatchev, Dual superconformal symmetry of scattering amplitudes in $N=4$ super-Yang-Mills theory, Nucl. Phys. B $\mathbf{8 2 8}$ (2010) 317 [arXiv:0807.1095] [INSPIRE].

[68] J.M. Drummond, J. Henn, G.P. Korchemsky and E. Sokatchev, Generalized unitarity for $N=4$ super-amplitudes, Nucl. Phys. B 869 (2013) 452 [arXiv:0808.0491] [InSPIRE].

[69] N. Arkani-Hamed, J.L. Bourjaily, F. Cachazo, A.B. Goncharov and A. Postnikov and J. Trnka, Scattering Amplitudes and the Positive Grassmannian, Cambridge University Press (2012) [DOI].

[70] F. Brown and C. Duhr, A double integral of dlog forms which is not polylogarithmic, (2020) [arXiv: 2006. 09413] [INSPIRE].

[71] K.-T. Chen, Algebras of Iterated Path Integrals and Fundamental Groups, Trans. Am. Math. Soc. 156 (1971) 359.

[72] K.-T. Chen, Iterated path integrals, Bull. Am. Math. Soc. 83 (1977) 831 [inSPIRE].

[73] A. Goncharov, Geometry of Configurations, Polylogarithms, and Motivic Cohomology, Adv. Math. 114 (1995) 197,. 
[74] A.B. Goncharov, Multiple polylogarithms, cyclotomy and modular complexes, Math. Res. Lett. 5 (1998) 497 [arXiv: 1105.2076] [INSPIRE].

[75] A.B. Goncharov, Multiple polylogarithms and mixed Tate motives, math/0103059 [INSPIRE].

[76] A.B. Goncharov, Galois symmetries of fundamental groupoids and noncommutative geometry, Duke Math. J. 128 (2005) 209 [math/0208144] [INSPIRE].

[77] F. Brown, On the decomposition of motivic multiple zeta values, arXiv:1102.1310 [INSPIRE].

[78] C. Duhr, Hopf algebras, coproducts and symbols: an application to Higgs boson amplitudes, JHEP 08 (2012) 043 [arXiv: 1203.0454] [INSPIRE].

[79] V. Del Duca, C. Duhr and V.A. Smirnov, An Analytic Result for the Two-Loop Hexagon Wilson Loop in $N=4$ SYM, JHEP 03 (2010) 099 [arXiv:0911.5332] [INSPIRE].

[80] V. Del Duca, C. Duhr and V.A. Smirnov, The Two-Loop Hexagon Wilson Loop in $N=4$ SYM, JHEP 05 (2010) 084 [arXiv: 1003.1702] [INSPIRE].

[81] V. Del Duca, C. Duhr and V.A. Smirnov, The massless hexagon integral in $D=6$ dimensions, Phys. Lett. B 703 (2011) 363 [arXiv:1104.2781] [INSPIRE].

[82] L.J. Dixon, J.M. Drummond and J.M. Henn, The one-loop six-dimensional hexagon integral and its relation to MHV amplitudes in $N=4$ SYM, JHEP 06 (2011) 100 [arXiv:1104.2787] [INSPIRE].

[83] SpaSM: a Sparse direct Solver Modulo p, The SpaSM group, v1.2 (2017)

[http://github.com/cbouilla/spasm].

[84] F.C.S. Brown, Multiple zeta values and periods of moduli spaces $M 0, n$ ( $R$ ), Annales Sci. Ecole Norm. Sup. 42 (2009) 371 [math/0606419] [INSPIRE].

[85] E. Panzer, Feynman integrals and hyperlogarithms, Ph.D. thesis, Humboldt U., 2015. arXiv: 1506.07243. 10.18452/17157 [INSPIRE].

[86] J. Blumlein, D.J. Broadhurst and J.A.M. Vermaseren, The Multiple Zeta Value Data Mine, Comput. Phys. Commun. 181 (2010) 582 [arXiv:0907.2557] [InSPIRE].

[87] N. Beisert, B. Eden and M. Staudacher, Transcendentality and Crossing, J. Stat. Mech. 0701 (2007) P01021 [hep-th/0610251] [INSPIRE].

[88] L. Dixon and F. Dulat, to appear.

[89] L. Dixon, private communication. 